Estimating New Quantities from Longitudinal Test Scores to Improve Forecasts of Future Performance

Daniel McNeish ${ }^{1}$, Denis G. Dumas ${ }^{2}, \&$ Kevin J. Grimm ${ }^{1}$

${ }^{1}$ Arizona State University, USA

${ }^{2}$ University of Denver, USA

Contact Information:

Daniel McNeish, PO Box 871104, Arizona State University, Tempe, AZ 85287. Email: dmcneish@asu.edu 


\begin{abstract}
Psychometric models for longitudinal test scores typically estimate quantities associated with single-administration tests, like ability at each time-point. However, models for longitudinal tests have not considered opportunities to estimate new quantities that are unavailable from singleadministration tests. Specifically, we discuss dynamic measurement models - which combine aspects of longitudinal IRT, nonlinear growth models, and dynamic assessment - to directly estimate capacity, defined as the expected future score once the construct has fully developed. After discussing the history and connecting these areas into a single framework, we apply the model to verbal test scores from the Intergenerational Studies, which follow 494 people from 3 to 72 years old. The goal is to predict adult verbal scores (Age $\geq 34$ ) from adolescent scores (Age $\leq 20)$. We held-out the adult data for prediction and compared predictions from traditional longitudinal IRT ability scores and proposed dynamic measurement capacity scores from models fit to the adolescent data. Results showed that the $R^{2}$ from capacity scores were 2.5 times larger than the $R^{2}$ from longitudinal IRT ability scores (43\% vs. 16\%), providing some evidence that exploring new quantities available from longitudinal testing could be worthwhile when an interest in testing is forecasting future performance.
\end{abstract}




\section{Estimating New Quantities from Longitudinal Test Scores to Improve Forecasts of Future Performance}

In North America, the history of large-scale, high-stakes psychometrics goes back to

World War I, with a goal to evaluate cognitive abilities of military recruits in order to sort them into war-related positions (Terman, 1918; Thorndike, 1921). The methodological rigor of these initial attempts in the infancy of psychometrics pale in comparison to the state of psychometrics today. Since these early testing programs, the statistical and psychometric literature has made substantial strides with respect to devising assessments with highly-vetted items that minimize measurement error and deliver more precise and reliable scores (Jones \& Thissen, 2006; Nicewander, 1993, Rust \& Golombok, 2009).

Despite this notable increase in the quality of psychometric work over the last century, some major areas for the improvement of psychometric research and practice remain. For example, historically, testing has had a principal interest in developed constructs that are fully developed at time of testing (e.g., Carroll, 1993). For instance, a test of tenth-grade reading given to a tenth-grade student would test a developed construct. Tests of developed constructs are typically single-administration whereby the test is administered one time (Sternberg \& Grigorenko, 2002). However, as psychometric endeavors expanded, interests in more ambitious constructs grew to include developing constructs - constructs that are partially formed at the time of testing but that will not be fully realized until some point in the future. With developing constructs, multiple administrations are necessary to track progress towards the fully realized ability (Sternberg et al., 2002). However, single-administration tests are routinely used to assess developing constructs (e.g., Pfieffer, 2012), even though it falls outside the intended use of the test. In these circumstances, the construct at the time of testing is used to extrapolate what the fully realized ability will be. That is, the test is capturing what the student has achieved (the 
developed construct) rather than the more direct interest of a student's capacity (the developing construct) and doing so is rife with tenuous assumptions (Erwin \& Worrell, 2012; Vygotsky, 1934/1962).

Single-administration tests for capacity have been a magnet for criticism from the very beginnings of psychometrics as a field of study. W.E.B. du Bois criticized this practice directly in his 1920 essay Race Intelligence, arguing that current ability (the developed construct) could not be logically equated to capacity (the developing construct). He predicted that confounding developed constructs with developing constructs would inevitably be a tool for the continued oppression of those who have historically had fewer opportunities to develop their abilities early in life. This situation is especially true if those scoring poorly on single-administration tests never receive the instruction necessary to develop their ability (e.g., they are denied college admission or placed in a lower academic track), leading to a circular affirmation of the assessment's under-prediction of their potential. Edward Thorndike echoed this sentiment by stating, "Some of us, I fear, claimed a generality for our measures of status and a surety of inference from them to original capacity which it would be very hard to justify" (1921, p. 125).

Today, the benefits of multiple-administration assessment - whereby people are repeatedly tested over time, though not necessarily with the exact same items - have been noted and such data are routinely collected at multiple levels of government (US Department of Education, 2011) in compliance with relatively recent legislation (e.g., No Child Left Behind). Additionally, assessment programs from testing agencies, such as the ACT Aspire and NWEA's Measures of Academic Progress (MAP), track performance of students over time. ACT Aspire examines growth from Grade 3 to Grade 10 (ACT, 2014a, 2014b) and NWEA's MAP Growth assessments test students three times per year beginning in kindergarten and following them 
through $8^{\text {th }}$ grade, and in some cases through high-school (Thum \& Hauser, 2015; NWEA, 2019). Despite these policy changes emphasizing multiple-administration testing, longitudinal models have remained relatively distinct from psychometric models (Bauer \& Curran, 2015 p. 4). Analyses of test scores have expanded to incorporate longitudinal data (e.g., longitudinal IRT; e.g., Andrade \& Tavares, 2005) but these extensions focus on the same quantities produced by single-administration assessments, but simply estimates more of them (e.g., longitudinal IRT models estimate multiple abilities per student; for an exception, see the work of McArdle et al., 2009).

Such models are feasible and effective for their intended purpose, but as argued in McArdle et al. (2009) and as expanded in this paper, direct extensions miss opportunities to estimate new quantities that do not exist in single-administration assessment. That is, rather than generalizing the traditional single-administration framework to produce multiple ability scores per person as in longitudinal IRT, the multiple administrations afford opportunities to directly estimate different quantities - like the aforementioned capacity - that may be of more central psychological or educational interest. Specifically, we show that by blending the seldomcombined literatures on longitudinal and psychometric modeling, models can be parameterized to make the elusive concept of learning capacity discussed a century ago by pioneering psychometricians and social scientists directly estimable.

To outline the structure of this paper, we first review the literature on preliminary conceptualizations of how to measure capacity, for which formal statistical models were largely absent. We then discuss the conceptual congruence between these early approaches and modern nonlinear growth models, as well as how reparameterizations of these formal models may be useful to extend this earlier research to the large-scale assessment environment that operates 
today. To provide evidence that such an approach improves forecasting of future performance, we describe a motivating example that combines three separate lifespan studies to produce verbal ability scores across the lifespan from ages 3 to 72 . We separate the data into a training sample (age less than 20) and a holdout sample for prediction (the last time-point for each person) to mimic how standardized testing programs often operate (i.e., students are tested at the end of adolescence and the score is used to infer future performance). We then describe fitting our reparametrized models - referred to as Dynamic Measurement Models - and our methodology for comparing forecasts of late-lifespan verbal ability from direct capacity estimates and longitudinal IRT scores. Results show that the variance explained by direct capacity estimates from our proposed Dynamic Measurement Model is more than twice the variance explained from longitudinal IRT scores $\left(R^{2}=43 \%\right.$ vs. $\left.16 \%\right)$. Implications, limitations, and future directions are then discussed.

\section{Estimating Capacity}

\section{Preliminary Conceptualization of Capacity with Dynamic Assessment}

In the years immediately following World War II, Reuven Feuerstein worked in Israel to assess cognitive abilities of child survivors of concentration camps to sort them into grade level so they could continue their education (this work was eventually published in Feuerstein, Rand, \& Hoffman, 1979). However, they found that single-administration assessment systematically underestimated these students' appropriate grade level because their traumatic experiences meant that they knew less than would be otherwise expected for their age. That is, tests for developed constructs failed because these children had not been exposed to the concepts necessary for the test to be meaningfully used. Nonetheless, the children's capacity to acquire new knowledge (a developing construct) remained intact and was hypothesized to be a more relevant construct. 
To address this situation, Feuerstein developed a system called Dynamic Assessment wherein a student is tested multiple times with targeted learning opportunities between assessments. The implicit model of Dynamic Assessment is shown in Figure 1 - student learning and improvement is plotted over time to find the capacity - expected future score once the construct of interest has fully developed (Feuerstein, Feuerstein, \& Falik, 2015). Student learning typically follows a nonlinear, decelerating trajectory towards some asymptote with the asymptote reflecting an estimate of capacity. In Dynamic Assessment, this capacity is the quantity of interest rather than ability from any single assessment used to calculate it.

The development of Dynamic Assessment methods continues into the present with current research seeking to refine the most appropriate tasks, modes of instruction, and timescale of the test administrations in order to produce the most meaningful capacity scores (Elliott, Resing, \& Beckmann, 2018; Resing, Bakker, Pronk, \& Elliott, 2017). In some countries (e.g., the Netherlands, Israel), Dynamic Assessment is used as a method to produce estimates of capacity for test respondents from historically marginalized populations (Haywood \& Lidz, 2007), second language acquisition (Lantolf \& Poehner, 2011), or those who have an intellectual disability (McLaughlin \& Cascella, 2008) or who are intellectually gifted (Kirscherbaum, 1998).

[Figure 1 about here]

While the idea is a clever one to circumvent real world challenges, there are difficulties that can prevent Dynamic Assessment from being generalized to the large-scale testing programs that currently operate in North America and around the world. For instance, Dynamic Assessment is quite resource intensive in terms of time and finances given the necessarily close correspondence between a clinician and a student during the targeted learning opportunities between test occasions. This one-on-one approach to learning also makes standardization more 
difficult. In addition, Dynamic Assessment has historically not relied on formalized statistical models, but in its most common instantiation, features the descriptive plots of student growth with no underlying psychometric framework by which to evaluate the quality of student scores. Nonetheless, the underlying idea has many merits for how to conceptualize measurement of capacity, which we expand in the following sections.

Specifically, McNeish and Dumas (2017) noted that the theoretical diagram of Dynamic Assessment from Figure 1 largely resembles a nonlinear growth trajectory and that current multiple-administration testing (especially with vertical scaling ${ }^{1}$ ) follows the general protocol of Dynamic Assessment, just on a larger time scale and with the instruction taking place in schools, rather than one-on-one with a clinician. This connection led to Dynamic Measurement Modeling (DMM; Dumas \& McNeish, 2017), whose goal is to formalize the concept of Dynamic Assessment to measure capacity with a formal statistical model using multiple-administration test scores but without requiring the resource-intensive one-on-one interventions between test administrations as in Dynamic Assessment. Before covering DMM specifically, we first discuss reparameterizations of growth models because the core idea of using DMM for measuring capacity is rooted in this concept.

\section{Interpretable Parameterizations with Longitudinal Data}

As noted in the introduction, methods for multiple-administration assessment like longitudinal IRT retain a focus on the individual assessments and handle the multiple

\footnotetext{
${ }^{1}$ Vertical scaling is a method for scoring tests that places the scores of two or more tests onto a single scale when the tests are on the same topic but vary in difficulty. For example, math tests intended for $2^{\text {nd }}$ grade and $3^{\text {rd }}$ grade students might each have separate scale scores that range from 100 to 400 . This makes it easy to compare within a grade but makes comparisons across grades difficult (e.g., a 300 in $2^{\text {nd }}$ grade is not the same as a 300 in $3^{\text {rd }}$ grade). The tests can be vertically scaled so that scores from both tests are on a single scale, perhaps ranging from 100 to 500 (e.g., a score of 300 means the same thing whether the student is in $2^{\text {nd }}$ or $3^{\text {rd }}$ grade). By vertically scaling, it is possible to compare an advanced $2^{\text {nd }}$ grade student to students in $3^{\text {rd }}$ grade or inspect how a single student improved between $2^{\text {nd }}$ and $3^{\text {rd }}$ grade.
} 
administrations mostly by producing separate ability estimates at each time-point. In essence, this is a straight multivariate extension of single-administration testing. What tends to be less prominent in these methods is the consideration of what new quantities might be afforded with a more complex data structure and model reparameterization. That is, rather than providing repeated estimates of single-administration quantities, unique quantities might be available from multiple-administration assessment than are unavailable from single-administration quantities.

This is analogous to modeling longitudinal data in repeated measures ANOVA (or the general linear model framework, more broadly). Repeated measures ANOVA generalizes mean differences tests to determine whether the mean changes over time. What repeated measures ANOVA does not do is capitalize on the information in the repeated measures to yield more interpretable quantities. For instance, consider a hypothetical study with six repeated measures whose means are $0.84,2.02,1.30,3.12,2.90$, and 2.33. The left panel of Figure 2 shows a mean plot of the data, which bears a strong quadratic shape (increase to an apex, then descent thereafter). A sufficiently powered $F$ test from a repeated measures ANOVA would note that there is at least one pair of means that are unequal. A trend analysis would detect a quadratic trend such that $\hat{y}=.83+1.27 \times$ Time $-.19 \times$ Time $^{2}$ fits well through the data points. Though the general idea of a quadratic trend is simple enough, the exact interpretation is not so clear: simple and substantive meaningful questions like "what is the value of the outcome at the apex?" or "at what time does the apex occur?" are not easily determined.

As a benefit of the multiple measurement occasions, Cudeck and du Toit (2002) showed that the model can be reparametrized as $\hat{y}=\beta_{\text {Apex }}-\left(\beta_{\text {Apex }}-\beta_{0}\right)\left(\frac{\text { Time }}{\beta_{\text {ApexTime }}}-1\right)^{2}$ where $\beta_{0}$ is the intercept, $\beta_{\text {Apex }}$ is the value of the outcome $y$ at the apex, and $\beta_{\text {ApexTime }}$ is the time at which $\beta_{\text {Apex }}$ 
occurs. The right panel of Figure 2 shows the fit of the quadratic model; the model is the same as the model with time parameterized with additive polynomials (Time and Time ${ }^{2}$ ) but defines the curve with quantities of direct interest rather than polynomials of time with an undesirable interpretation (Preacher \& Hancock, 2015). Estimating this model yields the same intercept as the polynomial model of .83 but directly estimates the apex as 2.94 and estimates that the apex occurs at time 3.31 rather than giving the rate of change at Time $=0$ as 1.27 and the half the acceleration equal to -.19 (the interpretation obtained from a trend analysis following an ANOVA).

\section{[Figure 2 about Here]}

Extending this example to large scale or high-stakes assessment, a common goal is to extrapolate which students would most benefit from access to scarce resources such as access to specialized instruction or admission in selective universities. These types of decisions are largely made from extrapolating from the test scores themselves. However, with multiple administration assessment, rather than focusing on the individual time-points and directly extrapolating to the future, a curve can be fit through the multiple scores and the model can be parameterized such that each student's future capacity can be directly estimated, much like how the quadratic model above can be parameterized to directly estimate desired information like the apex. Specifics of this modeling framework are covered in the next section.

\section{Dynamic Measurement Models}

DMMs synthesize the ideas Cudeck and du Toit (2002), Feuerstein et al. (1979), McArdle et al. (2009), Preacher and Hancock (2015), and Vygotsky (1934/1962) by parameterizing models of multiple-administration test scores as a growth model with an upper asymptote to estimate capacity. That is, a growth curve is fit through test scores (provided that 
they are vertically scaled or on some other common metric) so that the asymptotes function as the capacity because, by definition, the upper asymptote serves as the estimated ability as time approaches infinity. The asymptote (and possibly other parameters) is then modeled with personspecific random effects as with standard mixed effects models. The general idea is that - with random effects - each student in the data receives a unique monotonic, decelerating growth curve fit through their test scores.

The model is parameterized so that an upper asymptote is directly estimated, which is on the same scale as the original test scores. Given that each student receives a unique growth curve, this means that each student has a potentially unique upper asymptote representing the abilitylevel they would be estimated to obtain once the construct were fully developed (e.g., as time approaches infinity) as well as a unique learning trajectory over time. As in Dynamic Assessment, this estimate of the upper asymptote serves as the capacity score. So, rather than extrapolating an ability score from one test administration into the future, DMM directly estimates the future capacity, given past scores and a parametric functional form that represents the students' learning trajectory (Dumas \& McNeish, 2017).

There are a variety of functional forms that can be parameterized to include an upper asymptote, each of which characterizes a different type of growth that may be differentially appropriate depending on the age of the students and researcher knowledge about how the construct of interest changes over time. Table 1 shows the functional form of six such trajectories and how they are parameterized to include an upper asymptote to estimate capacity. Figure 3 shows the different trajectories of learning that are best captured by each curve.

[Table 1 about here]

[Figure 3 about here] 
Essentially, the model is a generalization of standard latent variable models used to obtain ability scores, such as IRT or factor analysis (McNeish \& Dumas, 2018). In traditional psychometric models, items are administered to students and are scored to estimate students' ability. A response to any individual item is helpful but not completely sufficient for determining ability, but all the items together as a collective group are more informative and reliable for such a purpose (provided that all items are sufficiently related to the construct of interest). In DMM, the multiple-administration ability scores are themselves "scored" with a nonlinear growth model (where the upper asymptote serves as the capacity score) instead of the items. McNeish and Dumas (2018) describe capacity as a "meta-construct" because it scores the construct ability scores. The same logic between items and ability scores applies to ability scores and capacity any single ability score is not completely indicative of capacity by itself. However, treating the ability scores as a collective group can be informative for estimating capacity directly.

In the next section, we describe motivating example data, fitting DMMs to these data, and compare forecasts of verbal ability between DMM and longitudinal IRT.

\section{Motivating Data}

Our motivating data come from the Intergenerational Studies - three longitudinal lifespan studies of growth of cognitive abilities that began at the Institute of Human Development at the University of California, Berkeley beginning in the late 1920s and early 1930s: The Berkeley Growth study ( $N=67$; Bayley 1932, 1943, 1949), the Guidance-Control study $(N=226$; MacFarlane, 1939), and the Oakland Growth Study ( $N=201$; Jones, 1938, 1939a, 1939b). ${ }^{2}$ The Berkeley Growth Study enrolled infants born in 1928 or 1929 and were assessed annually from 4

\footnotetext{
${ }^{2}$ Anonymized data, SAS code, and R code used for the analysis are included on the first author's Open Science Framework page. An anonymous link to this page to preserve the blindness of peer review can be found here : https://osf.io/d49kb/?view_only=b79a8326578a45b5870bb15b2f118e9f
} 
to 18 years old and then at ages $21,26,36,52,66$, and 72 . The Guidance-Control Study recruited participants born in 1928 in Berkeley, California and assessments were given every 6 months between 2 and 4 years old, annually from 5 to 18 years old, and at age 40 and 52 . The Oakland Growth Study began in 1931 with children who were 10 to 12 years old (born between 1919 and 1921). Assessments were given every two years until age 18 and when participants were approximately 50 and 60 years old. Across all three studies, the total sample size for analysis was 494 with $50 \%$ of the sample being female. The social climate during the time of data collection resulted in the data being ethnically homogeneous. Additional detail on the design of each of these studies can be found in Grimm, McArdle, and Widaman (2011).

Many different assessments were given in each of the studies and our focus is on verbal ability tests. Verbal ability is of interest here because it is a crystalized ability that is expected to increase monotonically over the lifespan for healthy individuals until very late ages (Belsky, 1999) and is less susceptible to Flynn effects (e.g., Flynn 1987). This is unlike other abilities (e.g., memory or visuospatial) that would potentially be non-monotonic and could decline in the absence of repeated practice. Verbal ability was assessed in each study via either a StanfordBinet or Weschler intelligence test (or revisions thereof). These measures of verbal ability remain two of the top-ten most utilized psychometric tests in school systems around the world today (Oakland, Douglas, \& Kane, 2016).

Item-level data from the studies were linked across studies and across time using a longitudinal IRT model. There are multiple approaches for conducting longitudinal IRT when integrating different studies to create scores for subsequent use in growth models including single-group, multiple-group, multilevel, and multiple-group multilevel methods (Davoudzadeh, 2016). Single-group methods apply constraints across time to accommodate dependence over 
time, multiple-group methods explicitly model study-specific effects, multilevel methods explicitly model repeated measures, and multiple-group multilevel methods explicitly model both repeated measures and study-specific effects. Simulation results from Davoudzadeh (2016) suggest that the single-group approach - the simplest method, analytically - was no more biased than other methods with the added advantage of being the most efficient method for producing ability scores on the same metric across time and study (p. 39). Time-unstructured data can also be accommodated easily with this approach because it operates on a column vector of responses (e.g., "long" format data) rather than a row vector of responses (e.g., "wide" format data). The single-group approach has also been applied in McArdle et al. (2009) and Grimm et al. (2011), both of which used data from these same studies. Though most items were binary, there were some polytomous items with graded responses, so the scoring model was a partial credit model (Masters, 1982) such that

$$
\ln \left(\frac{P\left[x_{t m i}\right]}{1-P\left[x_{t m i}\right]}\right)=\theta_{t i}-\beta_{m}
$$

where $P\left[x_{t m i}\right]$ is the probability that the $i$ th person's response to item $m$ at time $t$ is in category $x$ (given that the response is either in category $x$ or category $x-1$ ), $\theta_{t i}$ is the unidimensional verbal ability estimate for the $i$ th person at time $t$, and $\beta_{m}$ is the item step difficulty for item $m$ which is assumed to be constant over time. The partial credit model does not include an item discrimination parameter and is considered part of the Rasch family.

Applying this model provides vertically scaled scores of verbal ability such that all scores are on a common scale across the lifespan. Figure 4 shows the verbal ability longitudinal IRT scores on this vertical scale for the 494 participants. As we discussed earlier, longitudinal IRT models are an excellent option to obtain multiple single-administration-type quantities like 
ability. However, as shown in the next section, curves can be fit to these multiple ability scores to estimate new quantities not available in single-administration assessment.

[Figure 4 about here]

\section{Fitted Dynamic Measurement Model}

In the current educational environment, assessments of cognitive abilities are commonly given to students through late adolescence and future ability or capacity is inferred from there (e.g., college admissions, job skills). To mimic this type of application, we restrict our data to scores obtained at age 20 or younger and fit a DMM to these ability scores to obtain capacity estimates for verbal ability in the age $\leq 20$ restricted sample. By doing so, we hold out the age > 20 data as a criterion for later prediction. Of the 494 people in the data, 486 people had at least one data point in the age $\leq 20$ sample.

DMM is a parametric model that fits a curve through multiple test scores, so the integrity of the capacity estimates is partially based on selecting a proper trajectory to characterize how scores change over time. To determine the best trajectory for verbal ability in our motivating data, we first fit the six curves from Table 1 with a marginal nonlinear regression model (i.e., with no random effects) using Proc Nlin in SAS 9.4. We compared the mean square error (MSE) for each of these curves to inform which would be the best candidates for inclusion in the full nonlinear mixed effect model, especially because these models are notorious for their difficulties with computation and convergence (e.g., Harring \& Liu, 2016).

The Michaelis-Menten model $(M S E=2.23)$, exponential model $(M S E=2.23)$, Weibull model $(M S E=2.19)$ and logistic model $(M S E=2.19)$ fit the best and had mean square errors that were close to one another, so we fit the full random effects version of each of these models in Proc Nlmixed in SAS 9.4 using maximum likelihood estimation via a first-order algorithm 
(Beal \& Sheiner, 1982) with double dogleg optimization (Dennis \& Mei, 1979; Gay, 1983) and a gradient convergence criteria of 1e-3.

\section{Michaelis-Menten}

We started with a model that placed random effects on all three growth parameters in the Michaelis-Menten model (intercept, capacity, and midpoint; see Table 1) and allowed all random effects to covary with each other. This model did not converge, as the estimated variance of the midpoint parameter was negative. Changing estimation and optimization methods did not resolve the issue, likely because the midpoint was near the end of the restricted data's observation window and was rather unstable. Reducing the dimensionality of the random effects covariance matrix often helps convergence (e.g., Grimm, Ram, \& Estabrook, 2016), so we removed the random effect for the midpoint but kept the random effects for the intercept and capacity and allowed them to covary. This model converged without issue and was used as the final Michaelis-Menten model. Specifically, this model can be written as

$$
\begin{aligned}
& \text { Verbal }_{t i}=\beta_{0 i}+\frac{\left(\beta_{C i}-\beta_{0 i}\right)\left(A g e_{t i}-3\right)}{\left[\gamma_{M}+\left(A g e_{t i}-3\right)\right]}+\varepsilon_{t i}, \text { for Age } e_{t i} \leq 20 \\
& \beta_{0 i}=\gamma_{0}+u_{0 i} \\
& \beta_{C i}=\gamma_{C}+u_{C i}
\end{aligned}
$$

where

$$
\begin{aligned}
& \mathbf{u}_{i} \sim \operatorname{MVN}\left(\mathbf{0},\left[\begin{array}{cc}
\tau_{0} & \\
\tau_{C 0} & \tau_{C}
\end{array}\right]\right) \\
& \boldsymbol{\varepsilon}_{i} \sim \operatorname{MVN}\left(\mathbf{0}, \sigma^{2}\right)
\end{aligned}
$$

where Verbal $_{t i}$ is the verbal ability at time $t$ for person $i, \beta_{0 i}$ is the estimated verbal ability for person $i$ when Age $=3$ (the youngest in the sample), $\beta_{C i}$ is the estimated capacity for person $i$ as Age $\rightarrow \infty$, and $\gamma_{M}$ is a midpoint parameter that is the age (plus 3, due to centering) at which 
verbal ability is halfway between $\beta_{0 i}$ and $\beta_{C i}$. In Equation $3, \tau_{0}$ is the variance of the personspecific intercepts (i.e., $\left.\operatorname{Var}\left(\beta_{0 i}\right)\right), \tau_{C}$ is the variance of the person-specific capacities (i.e., $\left.\operatorname{Var}\left(\beta_{C i}\right)\right), \tau_{C 0}$ is the covariance between person-specific intercepts and slopes, and $\sigma^{2}$ is the residual variance which was modeled constant across time based on approximate uniform spread of the data across time in Figure 2. The BIC of this model was 5,774.

\section{Exponential}

As with the Michaelis-Menten model, we began with a model that placed random effects on all three growth parameters (intercept, capacity, and rate; see Table 1) and allowed all random effects to covary with each other. This model converged, but the estimated correlation between the capacities and rates was out of bounds and exceeded 1 . We removed the random effect for the rate, but kept the random effects for the intercept and capacity and allowed them to covary, which converged without issue. The final fitted exponential model can be written as

$$
\begin{aligned}
& \operatorname{Verbal}_{t i}=\beta_{0 i}+\left(\beta_{C i}-\beta_{0 i}\right)\left(1-e^{\left[\gamma_{R}-\left(\operatorname{Age}_{t i}-3\right)\right]}\right)+\varepsilon_{t i}, \text { for Age } e_{t i} \leq 20 \\
& \beta_{0 i}=\gamma_{0}+u_{0 i} \\
& \beta_{C i}=\gamma_{C}+u_{C i}
\end{aligned}
$$

where

$$
\begin{aligned}
& \mathbf{u}_{i} \sim \operatorname{MVN}\left(\mathbf{0},\left[\begin{array}{ll}
\tau_{0} & \\
\tau_{C 0} & \tau_{C}
\end{array}\right]\right) \\
& \boldsymbol{\varepsilon}_{i} \sim \operatorname{MVN}\left(\mathbf{0}, \sigma^{2}\right)
\end{aligned}
$$

where all parameters have the same definition as in Equation 2 and 3 except that $\gamma_{R}$ is a growth acceleration rate parameter rather than a midpoint as in Equation 2. The BIC of this model was 5,760 . 


\section{Weibull}

We began with a model that placed random effects on all four growth parameters (lower asymptote, capacity, rate, and inflection; see Table 1) and allowed all random effects to covary with each other. The model did not converge with admissible random effect correlations unless we removed the random effects for the rate and inflection parameters. The final fitted Weibull model can be written as

$$
\begin{aligned}
& \text { Verbal }_{t i}=\beta_{C i}+\left(\beta_{C i}-\beta_{L i}\right)\left(e^{-\left(\gamma_{R} A g e_{i i}\right)^{\gamma /}}\right)+\varepsilon_{t i}, \text { for Age } e_{t i} \leq 20 \\
& \beta_{L i}=\gamma_{L}+u_{L i} \\
& \beta_{C i}=\gamma_{C}+u_{C i}
\end{aligned}
$$

where

$$
\begin{aligned}
& \mathbf{u}_{i} \sim \operatorname{MVN}\left(\mathbf{0},\left[\begin{array}{ll}
\tau_{L} & \\
\tau_{C L} & \tau_{C}
\end{array}\right]\right) \\
& \boldsymbol{\varepsilon}_{i} \sim \operatorname{MVN}\left(\mathbf{0}, \sigma^{2}\right)
\end{aligned}
$$

Time must be larger than 0 for the Weibull model, so we did not center Age in this model. The Weibull model features a lower asymptote rather than an intercept parameter, but the two are essentially exchangeable for the time window in these data. The BIC of this model was 5,650.

\section{Logistic}

We began with a model that placed random effects on all four growth parameters (lower asymptote, capacity, rate, and midpoint; see Table 1) and allowed all random effects to covary with each other. This model did not converge as multiple random effect correlations exceeded an absolute value of 1 . We removed the random effect for the rate but one of the random effect correlations remained greater than 1 , so we also removed the random effect for the midpoint. The model with only random intercepts, random capacities, and a covariance between them converged without issue. The final fitted logistic model can be written as 


$$
\begin{aligned}
& \text { Verbal }_{t i}=\beta_{L i}+\frac{\left(\beta_{C i}-\beta_{L i}\right)}{\left[1+e^{-\gamma_{R}\left[\left(\operatorname{Age}_{i i}-3\right)-\gamma_{M}\right]}\right]}+\varepsilon_{t i}, \text { for Age } e_{t i} \leq 20 \\
& \beta_{L i}=\gamma_{L}+u_{L i} \\
& \beta_{C i}=\gamma_{C}+u_{C i}
\end{aligned}
$$

where

$$
\begin{aligned}
& \mathbf{u}_{i} \sim \operatorname{MVN}\left(\mathbf{0},\left[\begin{array}{ll}
\tau_{L} & \\
\tau_{C L} & \tau_{C}
\end{array}\right]\right) \\
& \boldsymbol{\varepsilon}_{i} \sim \operatorname{MVN}\left(\mathbf{0}, \sigma^{2}\right)
\end{aligned}
$$

where redundant parameters have the same definition as in other equations. The BIC of this model was 5,650.

\section{Nonlinear Mixed Model Estimates}

Table 2 shows the estimates from the three nonlinear mixed effects models described in Equations 2 through 9 in the previous sections. As hinted at in the reporting of model fit, the Michaelis-Menten and exponential models were extremely close, as were the Weibull and logistic models. If plotted, the marginal structures are so close that each pair cannot be distinguished from each other. Figure 5 plots the marginal trajectories against the age $\leq 20$ restricted data. Again, only two curves appear because of the aforementioned overlap. Nonetheless, even though the marginal curves are identical, the person-specific curves were different between the different models, so the capacity estimates were different across all four models.

[Table 2 about here]

[Figure 5 about here]

Based on the model fit information, the sigmoidal models (Weibull and logistic) appeared to fit the best based on the BIC. Between these two models, we preferred the logistic model based on its substantive direct interpretation of the midpoint parameter compared to the more 
indirect inflection parameter from the Weibull model. Therefore, we proceed with the logistic model in subsequent analyses. We realize that the decision about which curve to select necessarily contains some subjectivity, so we present results of subsequent analyses from the exponential, Michaelis-Menten, and Weibull models in the supplemental material, Appendices A through C.

\section{Forecast Analysis}

To assess the forecast of verbal ability from the DMM capacity estimates, we computed the empirical Bayes predictions of the person-specific random effects from the logistic model in Equations 8 and 9 and correlated these person-specific random effects with the last observed verbal ability score for each person that occurred after age 20 . Using the reliability formula for DMM capacities derived in McNeish and Dumas (2018), the marginal reliability of the logistic model capacities was 0.74 . A plot of conditional reliability across the range of predicted capacities is shown in Figure 6.

[Figure 6 about here]

Because the data extend over the entire lifespan, the last observed verbal ability was treated as the person's ultimate asymptotic verbal ability (referred to hereafter as "Asymptotic Ability") and the value to which the estimates were compared. Of the 486 people with data in the age $\leq 20$ restricted sample, 280 people also had at least one data point past age 20 . In other words, we fit the DMM to 486 people but the correlation analyses are based on a sample of 280 (the number of people with data before and after age 20). Asymptotic Ability occurred between ages 34-39 for $9 \%$ of these 280 people, between $40-49$ for $9 \%$, between $50-59$ for $49 \%$, between 60-69 for $27 \%$, and between $70-72$ for $6 \%$. 
We compared the correlation with Asymptotic Ability and DMM Capacity to the correlation between Asymptotic Ability and the last observed verbal ability longitudinal IRT score in the age $\leq 20$ sample (referred to hereafter as "Age 20 Longitudinal IRT Score", though note that it may be slightly before age 20 if the participant was not assessed at age 20). The correlation between these Asymptotic Ability and Age 20 Longitudinal IRT Score mimics how single-administration assessment operates: the observed value at the end of adolescence is typically used as the best prediction of the individual's capacity. As a discriminant validity check, we also correlated DMM Capacity with Age 20 Longitudinal IRT Score to ensure that they were not representing identical information. The ultimate goal of the analysis was to assess whether the DMM capacities estimated from the restricted age $\leq 20$ data provided better forecasts of Asymptotic Ability than forecasts Age 20 Longitudinal IRT Scores from the end of the same window.

\section{Forecast Analysis Results}

When running the analysis, we noted three influential outliers. Each of these observations were outliers with respect to abnormally low Age 20 Longitudinal IRT Scores. To best accommodate these outliers, we report the analysis four different ways: (a) Pearson correlations with the outliers removed, (b) Pearson correlations with the outliers included, (c) Spearman correlations with the outliers removed, and (d) Spearman correlations with the outliers included. Table 3 shows these results along with 95\% confidence intervals based on a Fisher transformation.

\section{[Table 3 about here]}

Regardless of correlation index or how outliers are treated, a notable general finding was that DMM Capacity is more highly correlated with Asymptotic Ability than was Age 20 
Longitudinal IRT Score. The correlations between DMM Capacity and Asymptotic Ability were consistently in the high .60s whereas the correlations with Age 20 Longitudinal IRT Score and Asymptotic Ability were in the $.40 \mathrm{~s}^{3}{ }^{3}$ Using the confidence interval approach of Zou (2007) for significance testing overlapping dependent correlations as featured in the cocor R Package (Diedenhofen \& Musch, 2015), the Pearson correlation between Asymptotic Ability and DMM Capacity was significantly different from the Pearson correlation between Asymptotic Ability and Age 20 Longitudinal IRT Score with outliers (95\% confidence interval for the difference: $[.17, .35])$ and without outliers (95\% confidence interval for the difference: $[.14, .31])$. Similar patterns held for the Spearman correlations with outliers (95\% confidence interval for the difference: $[.09, .26])$ or without outliers (95\% confidence interval for the difference: $[.07, .27])$.

The picture becomes starker if linear regression is used to compute $R^{2}$ variance explained measures for the predictions. Table 4 shows the $R^{2}$ values for Asymptotic Ability regressed on DMM Capacity or Age 20 Longitudinal IRT Score along with 95\% confidence intervals. Figure 7 plots the regression lines from the two different models. From Table 4, it can be seen that DMM Capacity accounts for more than twice as much variance in Asymptotic Ability as does Age 20 Longitudinal IRT Score. These notable differences demonstrate the advantages of explicitly modeling the capacity as a parameter in DMM - longitudinal IRT has no such parameter and instead extrapolates from a single time-point, requiring somewhat unrealistic assumptions concerning growth in the intervening period of time.

[Table 4 about here]

[Figure 7 about here]

\footnotetext{
${ }^{3}$ These correlations are quite close for the models featuring other types of growth trajectories. The MichaelisMenten DMM capacity correlations with Asymptotic ability were the largest and exceeded .70 in some analyses. See the supplemental material for complete results.
} 


\section{Sensitivity Analysis}

Results in the previous section used age 20 as the cut-off given that this age tends to be a common interest considering that it falls near the transition into adulthood. To demonstrate that the same basic pattern of results holds, we re-ran the forecast analysis using three additional age cut-offs: 12 years, 15 years, and 30 years. Table 5 shows the same correlational analyses as shown in Table 3 across these different age cut-offs in comparison to the age 20 cut-off used in the main analysis. For brevity, Table 5 only includes the Pearson correlations with outliers included (comparable to the leftmost column containing correlations in Table 3). Other correlation coefficients for each of these additional age cut-offs can be found in the supplemental material, Appendix D.

\section{[Table 5 about here]}

Because verbal ability development begins to flatten around age 20 (as seen in the full data in Figure 4), DMM has more difficulty with the earlier cut-offs because these scores exhibit less nonlinearity because verbal ability is still ascending rapidly. As a result, (a) the correlation between DMM Capacity and Asymptotic Ability is lower for earlier cut-offs, (b) the reliability of the DMM capacities is lower with earlier cut-offs, and (c) the correlation between the longitudinal IRT scores and the DMM capacities is higher because the DMM Capacities lean more towards rank-order preservation (as assumed by extrapolation from Longitudinal IRT) in the absence of information near the curvature in the trajectory. As noted in McNeish \& Dumas (2017), DMM is most effective when there is data near the point of steepest curvature - Table 5 demonstrates this empirically. The DMM Capacity reliability and DMM Capacity forecasts improve with later cut-offs as data near the bend in the trajectory are uncovered. Nonetheless, 
even though the gap between the methods decreases, the DMM Capacity correlation with Asymptotic Ability remains significantly higher compared to longitudinal IRT.

\section{Practical Recommendations}

This section discusses practical suggestions for fitting DMMs or for designing studies intended for use with DMMs. Because DMMs operate in the nonlinear mixed effect model framework, sample size - both the number of people and the number of time-points - warrant consideration. With nonlinear trajectories, the number of time-points matters less than the location of the time-points. Timmons \& Preacher (2015) discuss how efficiency is highest when the densest concentration of time-points is near the trajectory's maximum curvature and that additional time-points distal from this location often add little information. Nonlinear mixed effects models also allow for time-unstructured data, so researchers need not collect data at the same time for each participant; in fact, varying the time of data collection may be beneficial for some applications where participant data is expensive, as it could help to better map out the entire curve with relatively few observations per person. Regarding the number of people, mixed effects models in general are susceptible to small sample estimation bias (Maas \& Hox, 2005) and the problem increases with model complexity (e.g., McNeish \& Stapleton, 2016). Though precise recommendations would depend on the number of random effects and the trajectory being fit, 50 participants would seem to be an approximate lower bound (McNeish, 2016). In terms of modeling, nonlinear mixed effects models are notoriously fickle and often exhibit convergence issues. This was observed in our analyses as we often had difficulty fitting models featuring random effects on all parameters while estimating all covariances. We would recommend that researchers not remove random effects for the capacity in any circumstance, as this parameter is the main focus of the analysis. Without random effects on the capacity, each 
person will not receive a unique estimate, which runs counter to spirit of the approach. Rate and midpoint parameters tend to be the most difficult to estimate with random effects and would be candidates for removal in the presence of estimation issues. Convergence problems related to random effects may be evaded by using a Cholesky decomposition of the random effect covariance matrix because this method tends to provide greater numerical stability (Kohli, Peralta, \& Bose, 2019). ${ }^{4}$ This is especially true when the variances of the random effects may have different magnitudes as would be common for models including rate parameters or when the outcome variable has a relatively narrow scale, as did the outcome used in our data. Another potential solution is to scale the rate parameter by multiplying the rate coefficient by a small number (e.g., .01) to make the scale of the estimated parameter larger to improve stability without altering its interpretation.

\section{Limitations}

First, DMM requires longitudinal ability scores to be on a common scale, with vertical scaling being a common way to obtain this in educational contexts. However, vertical scaling can be a difficult process (Briggs \& Weeks, 2009; Harris, 2007). Many methods have been developed and assessed with simulated data but the performance of these methods when applied to real-data is not always optimal (Tong \& Kolen, 2007). DMM heavily relies on each of the tests being on a common scale, so the extent to which the scores on the common scale are unreliable will undoubtedly permeate to the DMM parameter estimates (McNeish \& Dumas,

\footnotetext{
${ }^{4}$ Using a Cholesky decomposition of the random effect covariance matrix, the logistic model with all four random effects did converge (Cholesky decompositions did not converge for any other trajectory with all random effects). The fit was slightly better (BIC $=5,537$ vs. 5,650 ) and the marginal reliability of the capacities was a little higher (.80 vs. .74). Nonetheless, we proceeded with the version with two random effects (a) to better compare the different competing trajectories, which could only be fit with two random effects and (b) to better contextualize the ensuing sensitivity analysis because the Cholesky decomposition did not converge with different cut-offs, which required fitting models with only two random effects. The results were not appreciably different with a Cholesky decomposition and these results are reported in Appendix E of supplemental material.
} 
2017). In some traditional applications of dynamic assessment that complete testing measurement over a short time-span (i.e., hours, not years), the exact same test is administered to students at every time-point as a way to bypass the issue of vertical scaling (Haywood \& Lidz, 2007). However, over a longer time-span, such an expedient methodological choice is not possible, so vertical scaling may play a more prominent role in the ultimate utility of DMM. Second, our forecast analysis showed that the DMM capacities correlated better with scores from later in the lifespan than did Age 20 Longitudinal IRT Scores. However, the DMM capacities from some models can have scaling issues. In Table 2 , it can be seen that the Michaelis-Menten estimated the mean capacity to be in the mid-teens whereas the actual values later in the lifespan were mostly between about 2 and 6 . The same general issue occurred with the exponential model, though to a lesser extent. To be clear, the rank-order of the DMM capacities corresponded to late-lifespan well across all models and corresponded much better than the Age 20 Longitudinal IRT Scores. However, if the interest were in using the DMM values in an absolute sense rather than in a relative sense, this might be problematic, and for some trajectories more than others. For example, in a college admissions setting, this scaling issue would not be terribly relevant because the applicants would be compared to each other. However, if the interest were outside of educational settings and involved tests based on reaction speed that participants needed to eventually clear some threshold to be eligible (e.g., training of military pilots) and the capacity were used to estimate who might eventually be eligible in the future, the scaling issues may be problematic. McNeish and Dumas (2018) note that the scaling issues are most noticeable when the observation window is far from the asymptotic behavior of the outcome, which is especially noticeable in our motivating example where most scores are rapidly increasing prior to age 20 . 
Lastly, it is possible to infer from our use of the term "capacity" that we are claiming to measure an innate attribute, which is not the intention nor the result. We adopt the term capacity here from its historical use to describe the future realization of an ability; however, capacity as we use the word in association with DMM can be (and in an educational context, ideally should be) malleable. That is, capacity in the DMM context is an estimate of the upper limit of a curve of test scores. Individuals would certainly be able to move their capacity over time should they demonstrate improvement in the test scores underlying the DMM learning curve, coinciding with the main tenets of Feuersteinian and Vygotskyian theories of intelligence and learning upon which the principles of dynamic assessment rest. Preliminary work on DMM capacities from standardized math and reading tests up to eighth grade shows that DMM capacities tend to be much less related to demographic factors like socioeconomic status (Dumas \& McNeish, 2017, 2018); however, the effect was certainly not reduced to zero.

\section{Discussion}

As one might expect, results from the motivating data indicate that incorporating multiple test scores vastly improves forecasts of future performance compared to a single score. The contribution of DMM is not the insight that more information leads to better predictions, as this conclusion is rather intuitive. Instead, the contribution of DMM lies in how to incorporate and combine multiple test scores together to produce a single, interpretable score to capitalize on this axiom. That is, rather than using all the information to produce multiple ability scores (via longitudinal item response theory) whose interpretation is similar to if the test were only administered at that time point, DMM puts a functional form on top of multiple test administrations to produce a unique quantity not directly available in single-administration methods but that has been discussed and sought for several decades. Granted, capacity is not 
necessarily the interest of all tests - sometimes a single-administration score of a developed construct is perfectly suitable for particular testing goals (e.g., a summative grade-level knowledge test). However, such single-administration tests are not designed and are not able to extrapolate to capacity and are invalid for such a purpose.

Psychometric and statistical literatures have focused on extending single-administration quantities to multiple-administration data, rather than exploring new quantities afforded by the growing amount of multiple administration data. The advantages of considering all test administrations as a cohesive set rather than as multiple individual assessments has not yet been fully realized in the psychometric modeling literature. By embellishing psychometric models so that they capitalize on additional features of multiple-administration data — rather than treating them as a multivariate embodiment of the single-administration paradigm - forecasting test scores to future performance can be more accurate and the interpretations of these scores can be more refined, which is particularly desirable given the substantial personal and societal consequences that result from test score interpretations.

As outlined here, Dynamic Measurement Models are one possible way to score multipleadministration test data to directly estimate capacity by blending concepts from the growth modeling into the psychometric literature. Seeing as dynamic measurement is a nascent concept, further work is undoubtedly required to fully demonstrate its capabilities and ultimately uncover its weaknesses in order to more completely evaluate its potential contribution and whether capacity is a useful quantity for stakeholders. As testing policy and practice continues to more widely adopt multiple-administrations, models that incorporates new quantities available from longitudinal data will be indispensable for improving the inferences we make about students, whether or not future models follow the dynamic measurement framework presented here. 


\section{References}

ACT (2014a). ACT Aspire Summative Assessment Technical Bulletin \#1. Iowa City, IA: ACT. ACT(2014b). ACT Aspire Summative Assessment Technical Bulletin \#2. Iowa City, IA: ACT.

Andrade, D. F., \& Tavares, H. R. (2005). Item response theory for longitudinal data: population parameter estimation. Journal of Multivariate Analysis, 95, 1-22. http://dx.doi.org/10.1016/j.jmva.2004.07.005

Bauer, D. J. \& Curran, P. J. (2016). The discrepancy between measurement and modeling in longitudinal data analysis. In J. R. Harring, L. M. Stapleton \& S. N. Beretvas (Eds.), Advances in Multilevel Modeling for Educational Research (pp. 3-38). Charlotte, NC. Information Age Publishing.

Bayley, N. (1932). A study of the crying of infants during mental and physical tests. The Journal of Genetic Psychology, 40, 306-329. http://dx.doi.org/10.1080/08856559.1932.10532428

Bayley, N. (1943). Skeletal maturing in adolescence as a basis for determining percentage of completed growth. Child Development, 14, 1-46. http://dx.doi.org/10.2307/1125612

Bayley, N. (1949). Consistency and variability in the growth of intelligence from birth to eighteen years. The Journal of Genetic Psychology, 75, 165-196.

http://dx.doi.org/10.1080/00221325.1991.9914714

Beal, S. L., \& Sheiner, L. B. (1982). Estimating population kinetics. Critical Reviews in Biomedical Engineering, 8, 195-222.

Belsky, J. (1999). The psychology of aging: theory, research, and interventions. Pacific, CA: Brooks/Cole Publishing.

Briggs, D. C., \& Weeks, J. P. (2009). The impact of vertical scaling decisions on growth interpretations. Educational Measurement: Issues and Practice, 28, 3-14.

http://dx.doi.org/10.1111/j.1745-3992.2009.00158.x

Carroll, J. B. (1993). Human cognitive abilities: A survey of factor-analytic studies. New York: Cambridge University Press.

Cudeck, R., \& Du Toit, S. H. (2002). A version of quadratic regression with interpretable parameters. Multivariate Behavioral Research, 37, 501-519.

http://dx.doi.org/10.1207/S15327906MBR3704_04

Davoudzadeh, P. (2016). A Statistical Evaluation of Latent Variable Score Estimation from Simple and Complex Item Response Models: Implications for Integrative Data Analysis [Doctoral Dissertation]. University of California, Davis. 
Dennis, J. E. \& Mei, H.H.W. (1979). Two new unconstrained optimization algorithms which use function and gradient values. Journal of Optimization Theory and Applications, 28, 453-482. http://dx.doi.org/10.1007/BF00932218

Diedenhofen, B. \& Musch, J. (2015). cocor: A comprehensive solution for the statistical comparison of correlations. PloS One, 10, e0121945.

http://dx.doi.org/10.1371/journal.pone.0121945

DuBois, W.E.B. (2013). WEB DuBois on sociology and the Black community. Chicago: University of Chicago Press. Original work published 1920.

Dumas, D. G., \& McNeish, D. M. (2018). Increasing the consequential validity of reading assessment using dynamic measurement modeling: A comment on Dumas and McNeish (2017). Educational Researcher, 47, 612-614. http://dx.doi.org/10.3102/0013189X18797621

Dumas, D. G., \& McNeish, D. M. (2017). Dynamic measurement modeling: Using nonlinear growth models to estimate student learning capacity. Educational Researcher, 46, 284-292. http://dx.doi.org/10.3102/0013189X17725747

Elliott, J. G., Resing, W. C., \& Beckmann, J. F. (2018). Dynamic assessment: a case of unfulfilled potential?. Educational Review, 70, 7-17.

http://dx.doi.org/10.1080/00131911.2018.1396806

Erwin, J. O., \& Worrell, F. C. (2012). Assessment practices and the underrepresentation of minority students in gifted and talented education. Journal of Psychoeducational Assessment, 30, 74-87. http://dx.doi.org/10.1177/0734282911428197

Feuerstein, R., Feuerstein, R., Falik, L.H. (2015). Beyond smarter: Mediated learning and the brain's capacity for change. New York: Teachers College Press.

Feuerstein, R., Rand, Y., \& Hoffman, M. B. (1979). The dynamic assessment of retarded performers: the learning potential assessment device, theory, instruments, and techniques. Baltimore, MD: University Park Press.

Flynn, J. R. (1987). Massive IQ gains in 14 nations: What IQ tests really measure. Psychological Bulletin, 101, 171-191. http://dx.doi.org/10.1037/0033-2909.101.2.171

Gay, D.M. (1983). Subroutines for unconstrained minimization. ACM Transactions on Mathematical Software, 9, 503-524. https://doi.org/10.1145/356056.356066

Grimm, K. J., Mazza, G. L., \& Davoudzadeh, P. (2017). Model selection in finite mixture models: A k-fold cross-validation approach. Structural Equation Modeling, 24, 246-256. http://dx.doi.org/10.1080/10705511.2016.1250638

Grimm, K.J., McArdle, J. J., Widaman, K.F. (2011). Family-level variance in verbal ability change. In K. Trzesniewski, M. B. Donnellan, R. E. Lucas (Eds.), Secondary Data Analysis: An 
Introduction for Psychologists (pp. 2019-229). Washington, D.C.: American Psychological Association.

Grimm, K. J., Ram, N., \& Estabrook, R. (2016). Growth modeling: Structural equation and multilevel modeling approaches. New York: Guilford Publications.

Harring, J. R., \& Liu, J. (2016). A comparison of estimation methods for nonlinear mixed-effects models under model misspecification and data sparseness: A simulation study. Journal of Modern Applied Statistical Methods, 15, 27. http://dx.doi.org/10.22237/jmasm/1462076760

Harris, D. J. (2007). Practical issues in vertical scaling. In N.J. Dorans, M. Pommerich, \& P.W. Holland (Eds.). Linking and aligning scores and scales (pp. 233-251). New York: Springer.

Haywood, C \& Lidz, C. (2007). Dynamic assessment in practice: Clinical and educational applications. New York: Cambridge University Press.

Jones, H.E. (1938). The California adolescent growth study. The Journal of Educational Research, 31, 261-567. http://dx.doi.org/10.1080/00220671.1938.10880781

Jones, H.E. (1939a). The adolescent growth study: Procedures. Journal of Consulting Psychology, 3, 177-180. https://psycnet.apa.org/doi/10.1037/h0060864

Jones, H.E. (1939b). The adolescent growth study: Principles and methods. Journal of Consulting Psychology, 3, 157-159. https://psycnet.apa.org/doi/10.1037/h0050181

Jones, L. V., \& Thissen, D. (2007). A history and overview of psychometrics. In C. R. Rao \& S. Sinharay (Eds.), Psychometrics: Vol. 26. Handbook of statistics (pp. 1-28). Amsterdam: Elsevier

Kirschenbaum, R. J. (1998). Dynamic assessment and its use with underserved gifted and talented populations. Gifted Child Quarterly, 42, 140-147.

http://dx.doi.org/10.1177/001698629804200302

Kohli, N., Peralta, Y., \& Bose, M. (2019). Piecewise random-effects modeling software programs. Structural Equation Modeling, 26, 156-164.

http://dx.doi.org/10.1080/10705511.2018.1516507

Lantolf, J. P., \& Poehner, M. E. (2011). Dynamic assessment in the classroom: Vygotskian praxis for second language development. Language Teaching Research, 15, 11-33. http://dx.doi.org/10.1177/1362168810383328

MacFarlane, J. (1939). The guidance study, Sociometry, 2, 1-23. http://dx.doi.org/10.2307/2785296

Maas, C. J., \& Hox, J. J. (2005). Sufficient sample sizes for multilevel modeling. Methodology, 1, 86-92. http://dx.doi.org/10.1027/1614-2241.1.3.86

Masters, G. N. (1982). A Rasch model for partial credit scoring. Psychometrika, 47, 149-174. http://dx.doi.org/10.1007/BF02296272 
McArdle, J. J., Grimm, K. J., Hamagami, F., Bowles, R. P., \& Meredith, W. (2009). Modeling life-span growth curves of cognition using longitudinal data with multiple samples and changing scales of measurement. Psychological Methods, 14, 126-149. http://dx.doi.org/10.1037/a0015857

McLaughlin, K., \& Cascella, P. W. (2008). Eliciting a distal gesture via dynamic assessment among students with moderate to severe intellectual disability. Communication Disorders Quarterly, 29, 75-81. http://dx.doi.org/10.1177/1525740107311821

McNeish, D. (2016). Estimation methods for mixed logistic models with few clusters. Multivariate Behavioral Research, 51, 790-804.

http://dx.doi.org/10.1080/00273171.2016.1236237

McNeish, D., \& Dumas, D. (2018). Calculating conditional reliability for dynamic measurement model capacity estimates. Journal of Educational Measurement, 55, 614-634. http://dx.doi.org/10.1111/jedm.12195

McNeish, D., \& Dumas, D. (2017). Nonlinear growth models as measurement models: A second-order growth curve model for measuring potential. Multivariate Behavioral Research, 52, 61-85. http://dx.doi.org/10.1080/00273171.2016.1253451

McNeish, D., \& Stapleton, L. M. (2016). Modeling clustered data with very few clusters. Multivariate Behavioral Research, 51, 495-518. http://dx.doi.org/10.1080/00273171.2016.1167008

Nicewander, W.A (1993). Some relationships between the information function of IRT and the signal/noise ratio and reliability coefficient of classical test theory. Psychometrika, 58, 139-141. http://dx.doi.org/10.1007/BF02294477

NWEA. (2019). MAP ${ }^{\circledR}$ Growth ${ }^{\mathrm{TM}}$ Technical Report. Portland, OR: Author.

Oakland, T., Douglas, S., \& Kane, H. (2016). Top ten standardized tests used internationally with children and youth by school psychologists in 64 countries: A 24-year follow-up study. Journal of Psychoeducational Assessment, 34, 166-176. http://dx.doi.org/10.1177/0734282915595303

Olkin, I., \& Finn, J. D. (1995). Correlations redux. Psychological Bulletin, 118, 155-164. http://dx.doi.org/10.1037/0033-2909.118.1.155

Pfeiffer, S. I. (2012). Current perspectives on the identification and assessment of gifted students. Journal of Psychoeducational Assessment, 30, 3-9. http://dx.doi.org/10.1177/0734282911428192

Preacher, K. J., \& Hancock, G. R. (2015). Meaningful aspects of change as novel random coefficients: A general method for reparameterizing longitudinal models. Psychological Methods, 20, 84-101. http://dx.doi.org/10.1037/met0000028 
Ram, N., \& Grimm, K. J. (2009). Methods and measures: Growth mixture modeling: A method for identifying differences in longitudinal change among unobserved groups. International Journal of Behavioral Development, 33, 565-576. http://dx.doi.org/10.1177/0165025409343765

Resing, W. C., Bakker, M., Pronk, C. M., \& Elliott, J. G. (2017). Progression paths in children's problem solving: The influence of dynamic testing, initial variability, and working memory. Journal of Experimental Child Psychology, 153, 83-109. http://dx.doi.org/10.1016/j.jecp.2016.09.004

Rust, J., \& Golombok, S. (2009). Modern psychometrics: The science of psychological assessment. London: Routledge.

Sternberg, R.J. \& Grigorenko, E.L. (2002). Dynamic testing: The nature and measurement of learning potential. Cambridge, UK: Cambridge University Press.

Sternberg, R. J., Grigorenko, E. L., Ngorosho, D., Tantufuye, E., Mbise, A., Nokes, C., ... \& Bundy, D. A. (2002). Assessing intellectual potential in rural Tanzanian school children. Intelligence, 30, 141-162. http://dx.doi.org/10.1016/S0160-2896(01)00091-5

Terman, L.M. (1918). The use of intelligence tests in the army, Psychological Bulletin, 15, 177187. http://dx.doi.org/10.1037/h0071532

Thorndike, E.L. (1921). Intelligence and its measurement. Journal of Educational Psychology 12, 124-127. https://psycnet.apa.org/doi/10.1037/h0064596

Thum Y. M., \& Hauser, C. H. (2015). NWEA 2015 MAP Norms for Student and School Achievement Status and Growth. NWEA Research Report. Portland, OR: NWEA

Timmons, A. C., \& Preacher, K. J. (2015). The importance of temporal design: How do measurement intervals affect the accuracy and efficiency of parameter estimates in longitudinal research?. Multivariate Behavioral Research, 50, 41-55.

http://dx.doi.org/10.1080/00273171.2014.961056

Tong, Y., \& Kolen, M. J. (2007). Comparisons of methodologies and results in vertical scaling for educational achievement tests. Applied Measurement in Education, 20, 227-253.

http://dx.doi.org/10.1080/08957340701301207

US Department of Education (2011). Final report on the evaluation of the growth model pilot project. https://www2.ed.gov/rschstat/eval/disadv/growth-model-pilot/gmpp-final.pdf

Vygotsky, L. S. (1934/1962). Thought and language (E. Hanfmann \& G. Vakar, Trans.). Cambridge, MA: MIT Press.

Zou, G. Y. (2007). Toward using confidence intervals to compare correlations. Psychological Methods, 12, 399-413. http://dx.doi.org/10.1037/1082-989X.12.4.399 


\section{Table 1}

Overview of different growth trajectories that can be parameterized with an upper asymptote to model capacity

\begin{tabular}{|c|c|c|c|}
\hline Curve & Parameterization & Parameter Definitions & Notes \\
\hline Exponential & $\beta_{0}+\left(\beta_{C}-\beta_{0}\right)\left(1-e^{t \beta_{\text {Rate }}}\right)$ & $\begin{array}{ll}\beta_{0} & \text { Intercept } \\
\beta_{C} & \text { Capacity } \\
\beta_{R} & \text { Growth Rate } \\
t & \text { Time }\end{array}$ & \\
\hline Weibull & $\beta_{C}-\left(\beta_{C}-\beta_{L}\right)\left(e^{-\left(t \beta_{R}\right)^{\beta_{I}}}\right)$ & $\begin{array}{l}\beta_{L} \text { Lower Asymptote } \\
\beta_{C} \text { Capacity } \\
\beta_{R} \text { Growth Rate } \\
\beta_{I} \text { Inflection Point } \\
t \text { Time }\end{array}$ & $\begin{array}{l}\text { Exponential is a special case } \\
\text { where the inflection point is } 1 \text {. } \\
\text { The point of inflection is equal } \\
\text { to }\left(\frac{1}{\beta_{R}}\right)\left(\frac{\beta_{I}-1}{\beta_{I}}\right)^{\left(\frac{1}{\beta_{I}}\right)}\end{array}$ \\
\hline Michaelis-Menten & $\beta_{0}+\frac{\left(\beta_{C}-\beta_{0}\right) t}{\left(t+\beta_{M}\right)}$ & $\begin{array}{ll}\beta_{0} & \text { Intercept } \\
\beta_{C} & \text { Capacity } \\
\beta_{M} & \text { Midpoint } \\
t & \text { Time }\end{array}$ & $\begin{array}{l}\text { Midpoint is defined at the point } \\
\text { on the time scale where the } \\
\text { outcome is halfway between } \\
\text { intercept and capacity }\end{array}$ \\
\hline Logistic & $\beta_{L}+\frac{\left(\beta_{C}-\beta_{L}\right)}{\left[1+e^{-\beta_{R}\left(t-\beta_{M}\right)}\right]}$ & $\begin{array}{l}\beta_{L} \text { Lower Asymptote } \\
\beta_{C} \text { Capacity } \\
\beta_{R} \text { Slope at Midpoint } \\
\beta_{M} \text { Midpoint } \\
t \quad \text { Time }\end{array}$ & $\begin{array}{l}\text { Midpoint is defined at the point } \\
\text { on the time scale where the } \\
\text { outcome is halfway between } \\
\text { lower asymptote and capacity }\end{array}$ \\
\hline Gompertz & $\beta_{C} e^{\left[\ln \left(\frac{\beta_{0}}{\beta_{C}}\right) e^{\left(-t \beta_{R}\right)}\right]}$ & $\begin{array}{ll}\beta_{0} & \text { Intercept } \\
\beta_{C} & \text { Capacity } \\
\beta_{R} & \text { Growth Rate } \\
t & \text { Time }\end{array}$ & $\begin{array}{c}\text { The curve is fixed so that } 37 \% \\
\text { of growth occurs before the } \\
\text { inflection point }\end{array}$ \\
\hline Morgan-Mercer-Flodin & $\frac{\left(\beta_{0} \beta_{R}+\beta_{C} t^{\beta_{I}}\right)}{\left(\beta_{R}+t^{\beta_{I}}\right)}$ & $\begin{array}{ll}\beta_{0} & \text { Intercept } \\
\beta_{C} & \text { Capacity } \\
\beta_{M} & \text { Midpoint } \\
\beta_{I} & \text { Inflection Point } \\
t & \text { Time }\end{array}$ & $\begin{array}{l}\text { Michaelis-Menten is a special } \\
\text { case if the inflection parameter } \\
\text { is } 1 .\end{array}$ \\
\hline
\end{tabular}


Table 2

Comparison of full model estimates for Michaelis-Menten, exponential, and logistic trajectories

\begin{tabular}{cccccc}
\hline Parameter & Notation & Michaelis-Menten & Exponential & Logistic & Weibull \\
\hline Capacity & $\gamma_{C}$ & Fixed Effects & & & \\
Intercept & $\gamma_{0}$ & -8.67 & 6.76 & 2.24 & 2.02 \\
Rate & $\gamma_{R}$ & --- & -8.61 & -7.00 & -6.80 \\
Midpoint & $\gamma_{M}$ & 18.60 & -0.08 & 0.37 & 0.09 \\
Inflection & $\gamma_{I}$ & --- & --- & 6.43 & --- \\
\hline Capacity & Random Effect Variances & --- & --- & 2.79 \\
\hline Intercept & $\tau_{C}$ & 1.27 & 1.12 & 1.30 & 1.30 \\
\hline Intercept, Capacity & Corr $\left(u_{0}, u_{C}\right)$ & 1.22 & 1.22 & 1.51 & 1.49 \\
\hline BIC & \multicolumn{7}{c}{ Random Effect Correlations } & & & \\
\hline
\end{tabular}

Note: The intercept for the logistic and Weibull models is the lower asymptote, which functions similarly to an intercept given the observation window and centering in the data. 
Table 3

Correlations between DMM Capacity, Asymptotic Ability, and Age 20 Longitudinal IRT Score with $95 \%$ confidence intervals in parentheses

\begin{tabular}{lcccccc}
\hline & & \multicolumn{2}{c}{ Pearson } & \multicolumn{2}{c}{ Spearman } \\
\hline & & With & Without & With & Without \\
& & Outliers & Outliers & Outliers & Outliers \\
\hline Asymptotic Ability & DMM Capacity & .66 & .68 & .65 & .66 \\
& & $(.58, .72)$ & $(.61, .74)$ & $(.57, .71)$ & $(.59, .72)$ \\
Asymptotic Ability & Age 20 Longitudinal IRT Score & .40 & .46 & .48 & .49 \\
& & $(.30, .49)$ & $(.36, .55)$ & $(.38, .56)$ & $(.39, .57)$ \\
& & & & & \\
DMM Capacity & Age 20 Longitudinal IRT Score & .58 & .60 & .60 & .64 \\
& & & $(.52, .64)$ & $(.52, .67)$ & $(.54, .65)$ & $(.57, .71)$ \\
\hline
\end{tabular}


Table 4

$R^{2}$ variance explained measures for a linear regression of Asymptotic Ability on DMM Capacity or Age 20 Longitudinal IRT Score with $95 \%$ confidence interval in parentheses

\begin{tabular}{cccc}
\hline Outcome & Predictor & $\begin{array}{c}R^{2} \\
\text { With Outliers }\end{array}$ & $\begin{array}{c}R^{2} \\
\text { Without Outliers }\end{array}$ \\
\hline Asymptotic Ability & DMM Capacity & $\begin{array}{c}43 \% \\
(34 \%, 52 \%)\end{array}$ & $\begin{array}{c}47 \% \\
(39 \%, 56 \%)\end{array}$ \\
& Age 20 Longitudinal IRT Score & $\begin{array}{c}16 \% \\
(8 \%, 24 \%)\end{array}$ & $\begin{array}{c}21 \% \\
(13 \%, 30 \%)\end{array}$ \\
\hline \multicolumn{2}{c}{$\Delta R^{2}$} & $27 \%$ & $26 \%$ \\
\hline DMM Relative \% Increase & $169 \%$ & $124 \%$ \\
\hline
\end{tabular}

Note: confidence intervals for $R^{2}$ are calculated using the approximation from Olkin and Finn (1995) 
Table 5

Comparison of Pearson correlations with outliers and DMM Capacity Reliability using cut-off ages of $12,15,20$, and 30

\begin{tabular}{cccccc}
\hline & & \multicolumn{4}{c}{ Cut-Off Age } \\
\hline & & 12 & 15 & 20 & 30 \\
\hline Asymptotic Ability & DMM Capacity & .61 & .63 & .66 & .67 \\
& & $(.53, .68)$ & $(.58, .70)$ & $(.58, .72)$ & $(.59, .73)$ \\
Asymptotic Ability & Longitudinal IRT Score & .50 & .48 & .40 & .40 \\
& & $(.40, .58)$ & $(.38, .56)$ & $(.30, .49)$ & $(.30, .50)$ \\
DMM Capacity & Longitudinal IRT Score & .77 & .73 & .58 & .59 \\
& & $(.73, .80)$ & $(.68, .77)$ & $(.52, .64)$ & $(.52, .64)$ \\
\hline DMM Capacity Reliability & .61 & .69 & .74 & .75 \\
\hline
\end{tabular}

Note: the $95 \%$ confidence interval for the difference between the correlation of Asymptotic Ability with DMM and Longitudinal IRT does not include 0 using a cut-off of 12 years old (95\% $\mathrm{CI}=[.05, .18]), 15$ years old $(95 \% \mathrm{CI}=[.08, .22])$, or 30 years old $(95 \% \mathrm{CI}=[.18, .36])$ 


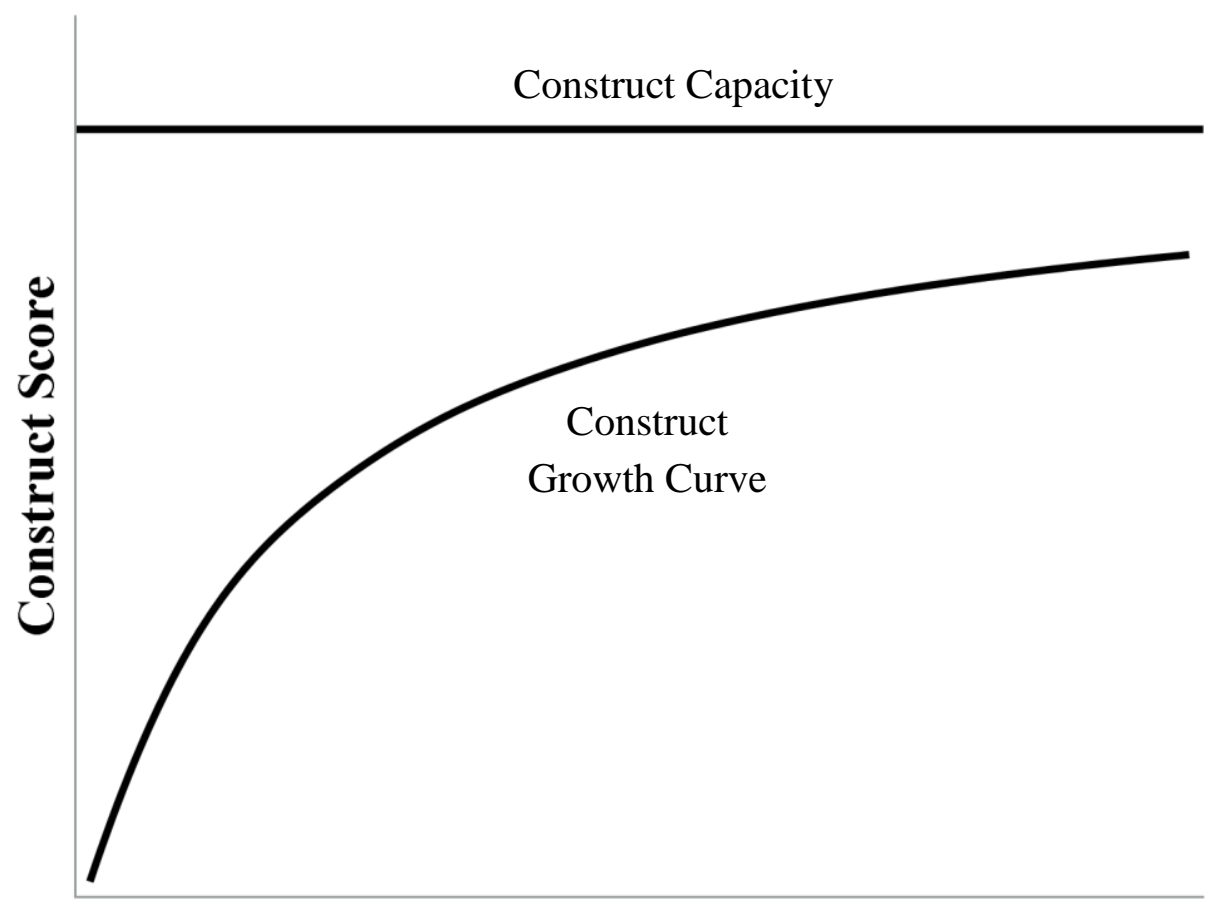

Time

Figure 1. Conceptual diagram of Dynamic Assessment 

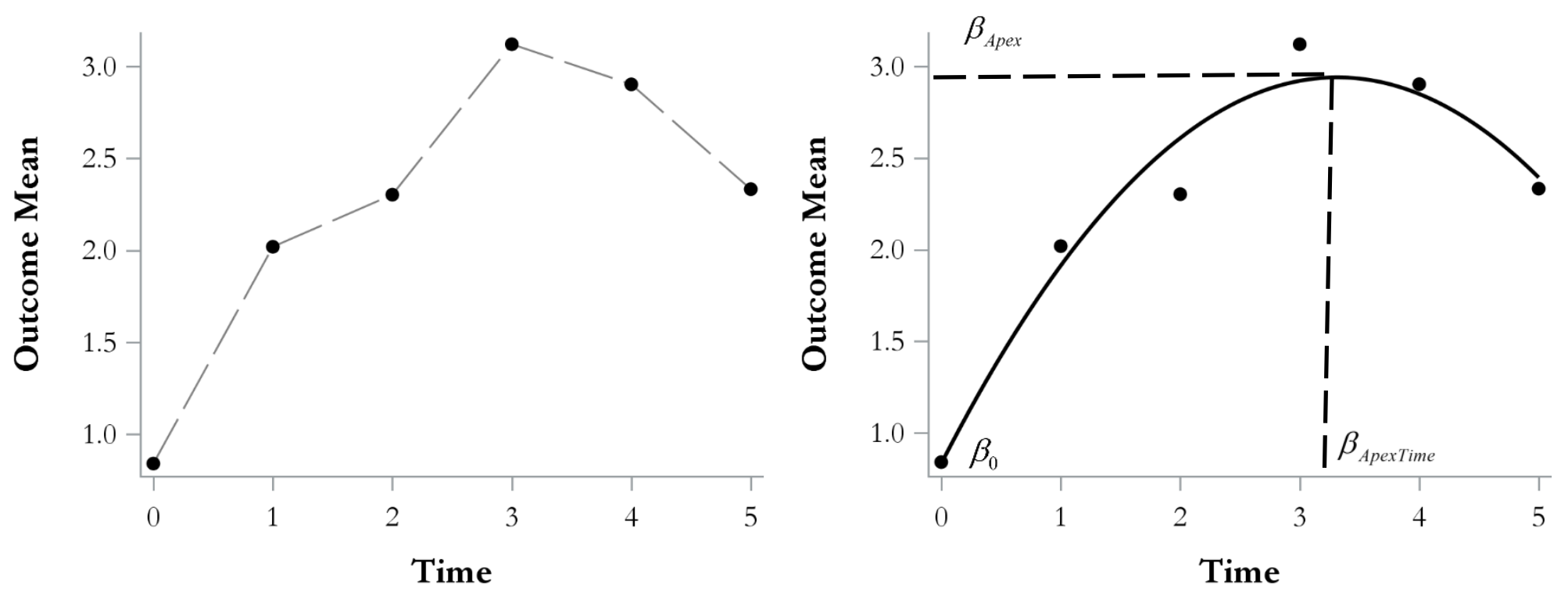

Figure 2. Comparison of mean differences with repeated measures ANOVA (left) and a quadratic model reparametrized as a nonlinear regression to yield the intercept, apex, and time of apex (right) 


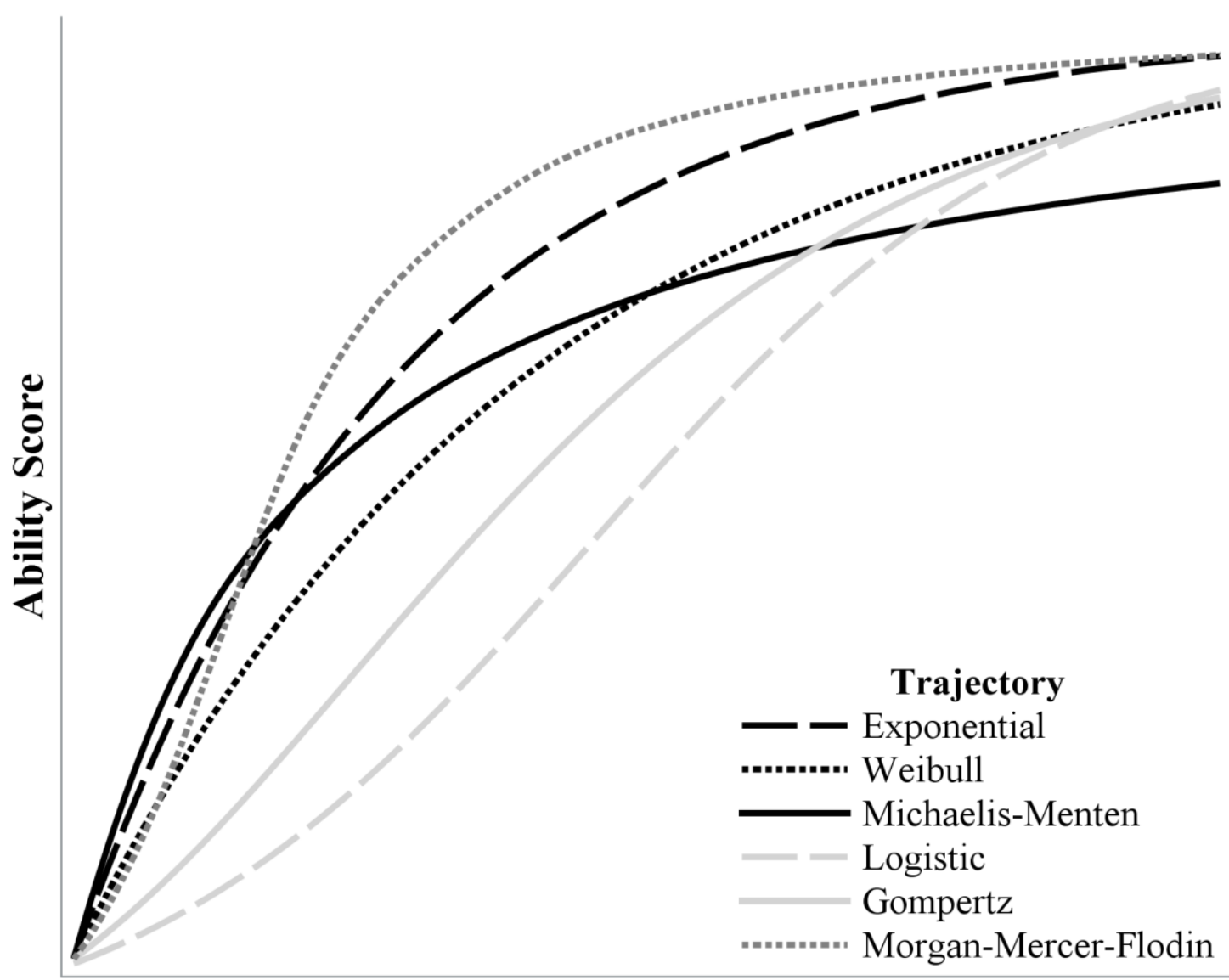

Time

Figure 3. Visual comparison of the shape of different curves parameterized with an upper asymptote to model capacity. All curves have the same intercept and upper asymptote, but model change between these two quantities differently. 


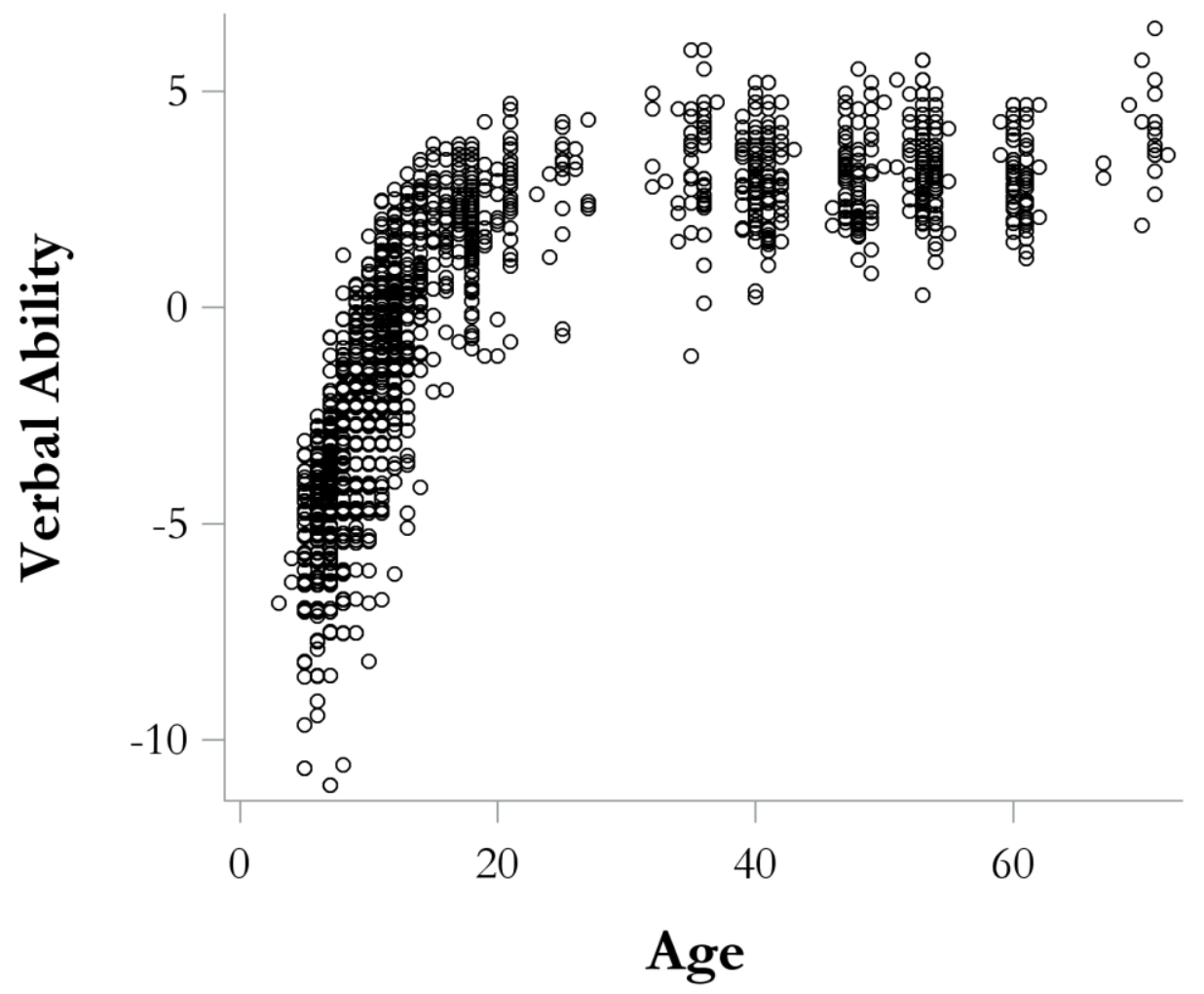

Figure 4. Scatterplot of test scores across the lifespan for all 494 participants. A monotonically increasing, but decelerating, growth trajectory appears to be present in these data. 


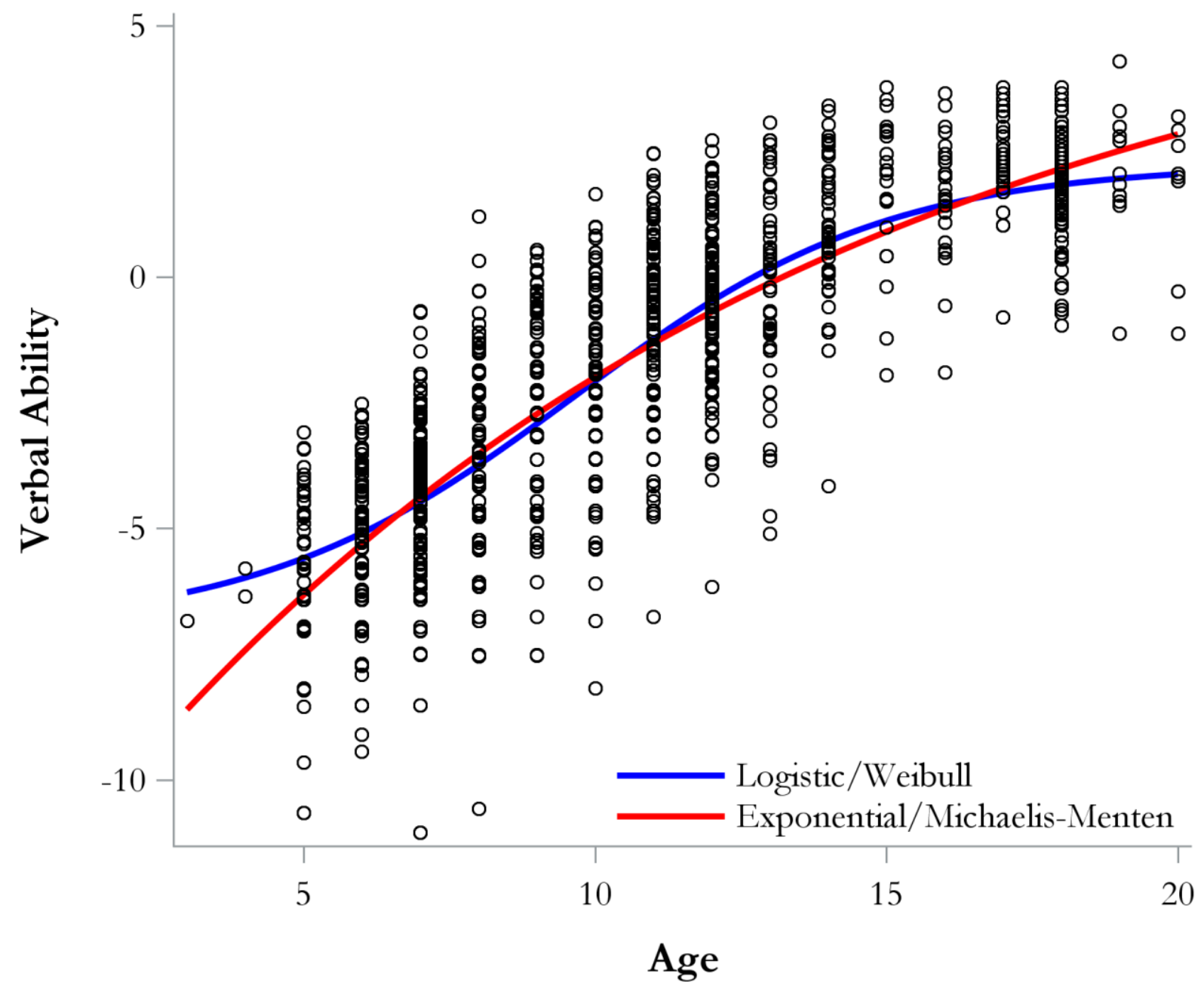

Figure 5. Scatter plot of Age $\leq 20$ restricted data with estimated mean trajectories superimposed in blue for the logistic and Weibull model and in red for the Michaelis-Menten and exponential model. The marginal structures were nearly identical for each pair, but the random effects were not. 


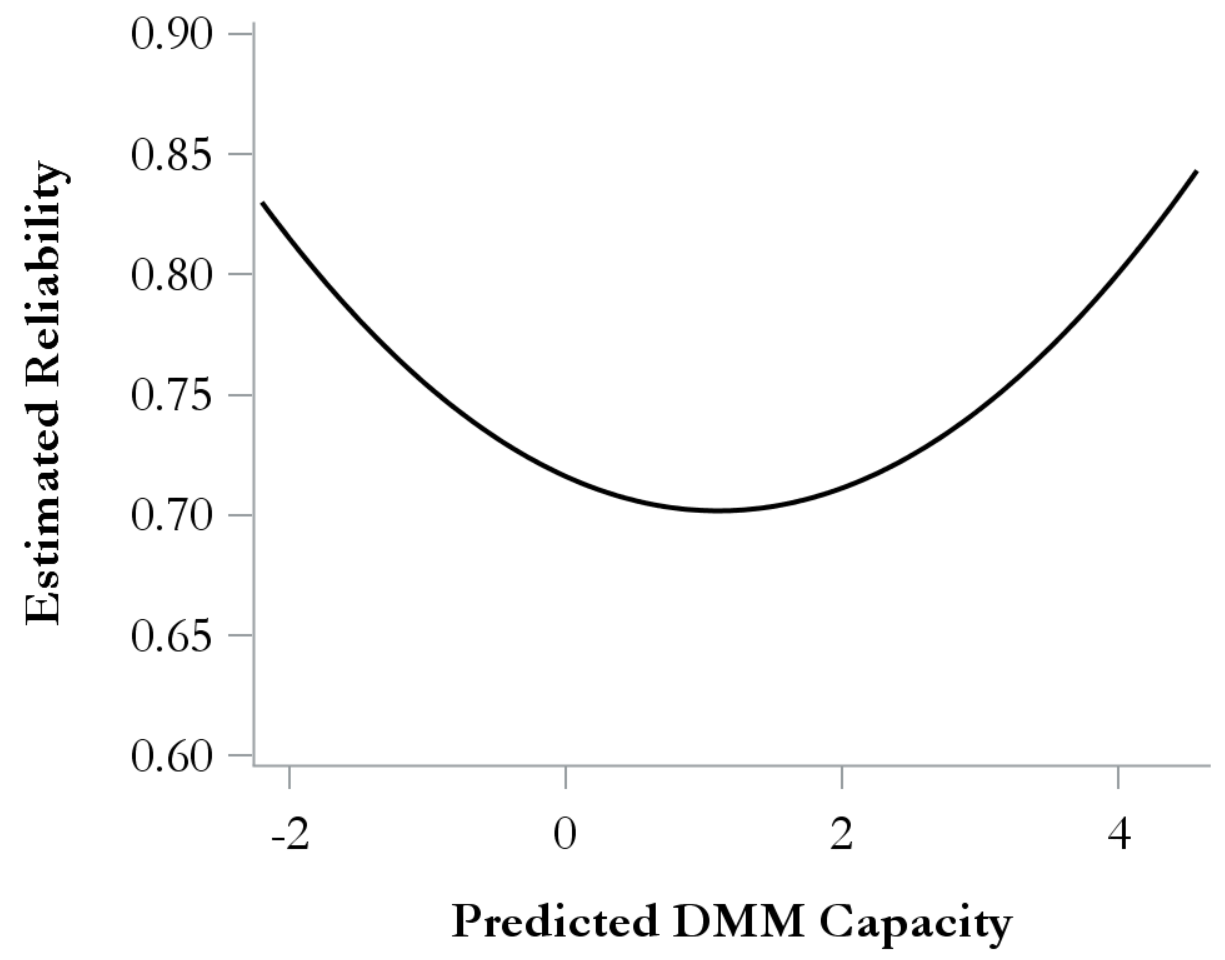

Figure 6. Smoothed conditional reliability plot for the estimated DMM capacities from the logistic model. 


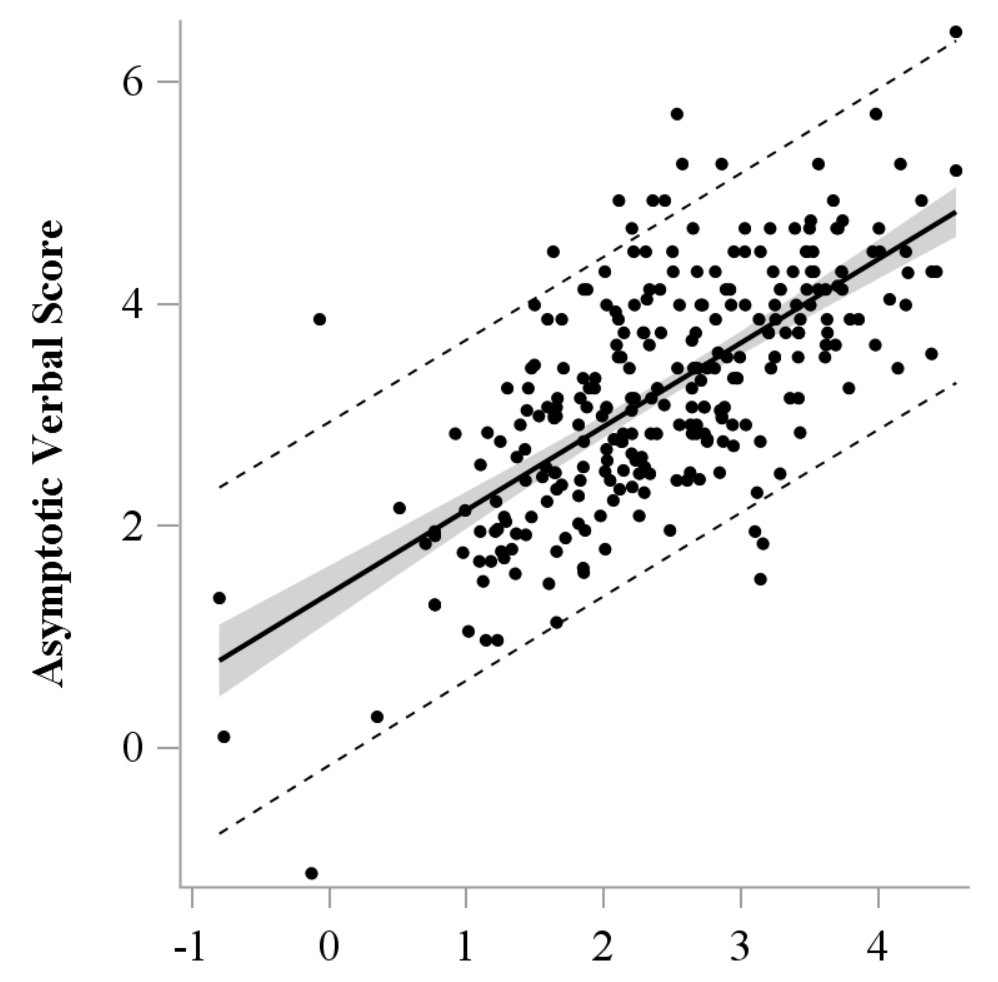

Dynamic Measurement Capacity

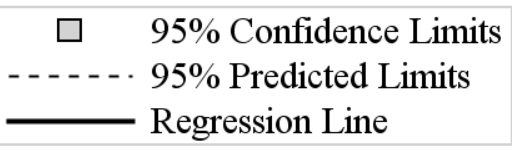

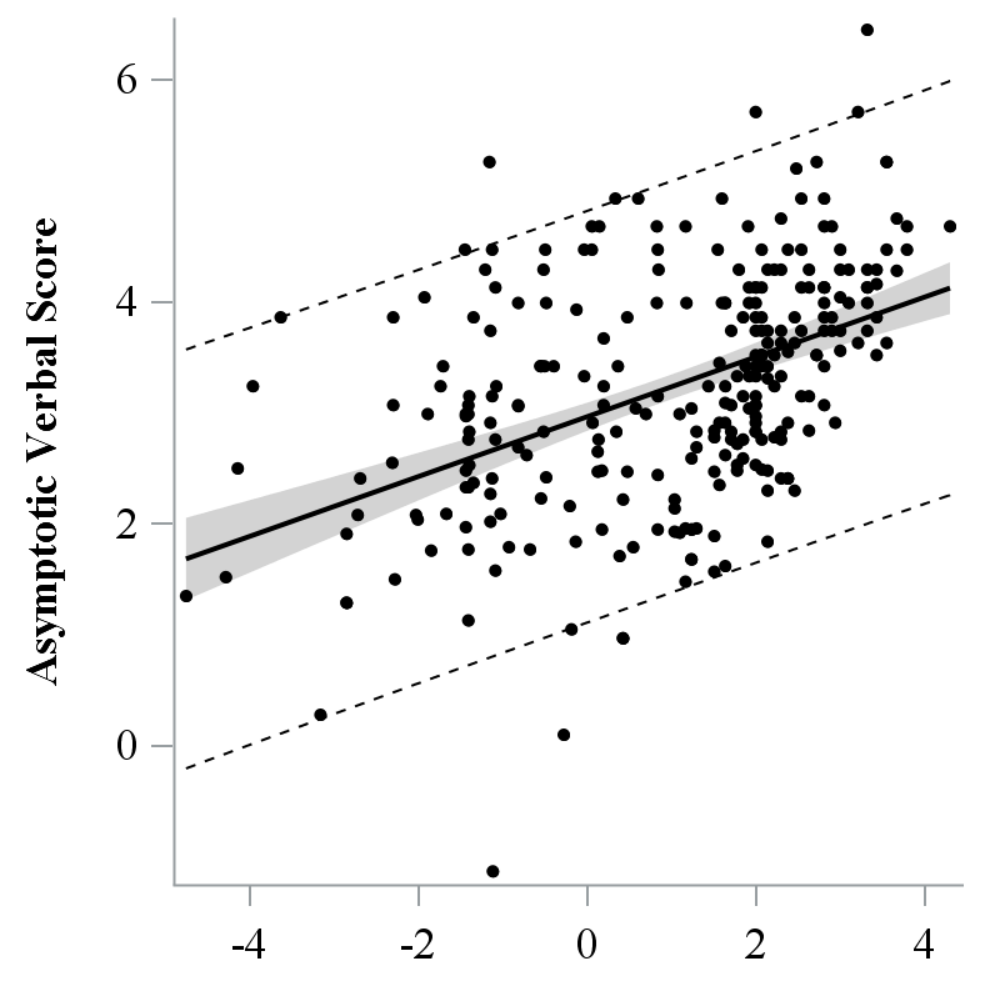

Age 20 Longitudinal IRT Score

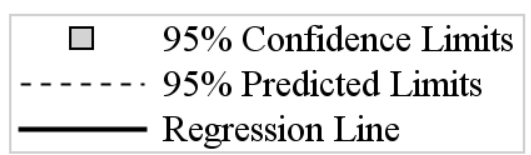

Figure 7. Comparison of fitted regression lines using the Asymptotic Ability in the data as predicted by DMM capacities (left) or Age 20 Longitudinal IRT Score (right) for the data without outliers. The plot on the left has more desirable forecasting qualities $\left(R^{2}=\right.$ $0.47, \mathrm{MSE}=.60)$ compared to the plot on the right $\left(R^{2}=0.16, \mathrm{MSE}=.88\right)$. 


\section{Supplemental Material:}

\section{Data Availability and Analysis Code:}

Anonymized data, SAS code, and R code used for the analysis are included on the first author's Open Science Framework page. An anonymous link to this page to preserve the blindness of peer review can be found here :

https://osf.io/d49kb/?view_only=b79a8326578a45b5870bb15b2f118e9f

The data are in the "long" format such that "v_ability" is the verbal ability score at a single timepoint. This verbal ability is on the vertical scale that resulted from the method of McArdle et al. (2009). Each person's data occupies multiple rows of the data, such that ID variables are repeated multiple times to indicate that multiple data points are collected for each person. The ID variables have been changed from the original data to because the original ID variables contained identifying information. The "Age" variable is the age at which the verbal ability score was collected.

The provided SAS code was run in Version 9.4 for the current analyses. SAS code for each of the different nonlinear trajectories is provided on the Open Science Framework page, linked above. Results from the trajectories not presented in the text are shown in this supplement.

The provide R code for significance testing dependent correlations was run in Version 3.4.1 of R. 


\section{Appendix A: Michaelis-Menten Trajectory}

\section{SAS Proc Nlmixed Code}

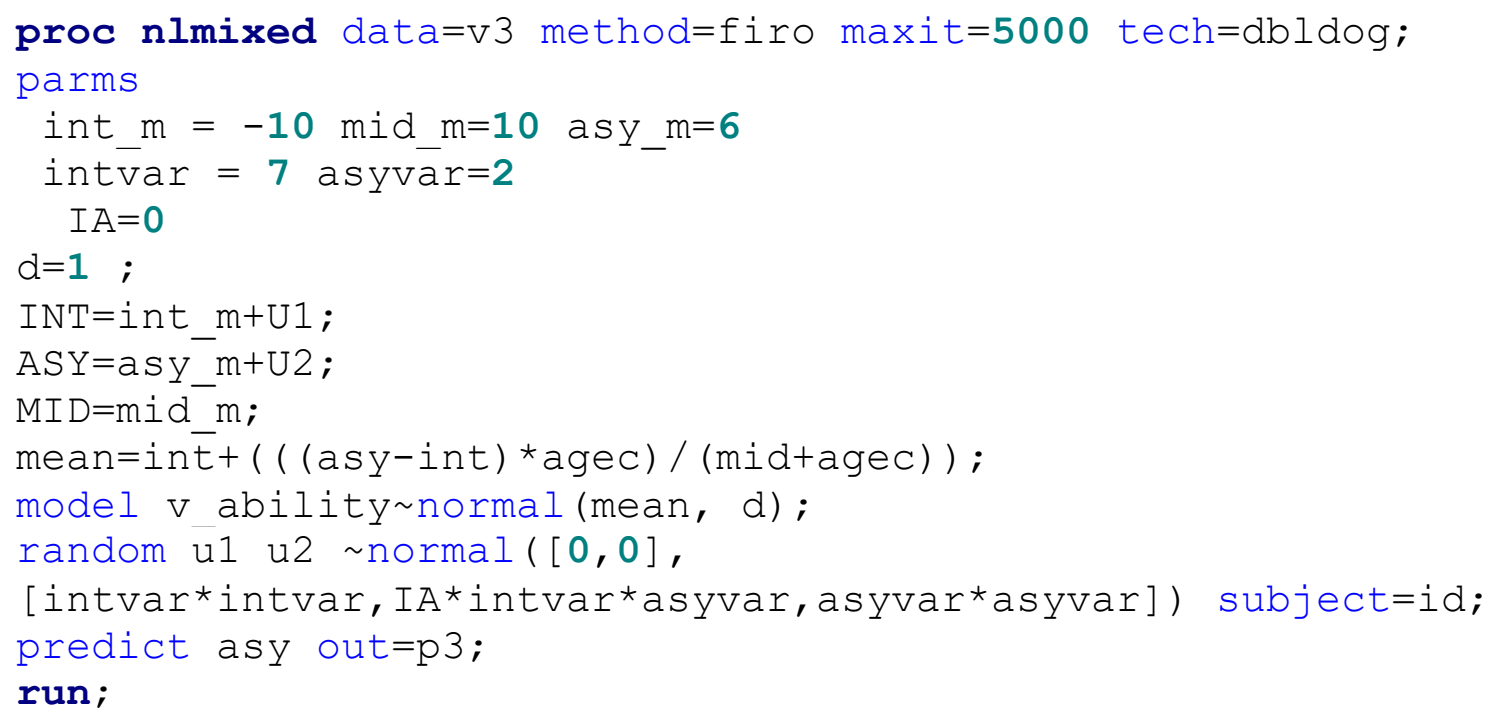

Table A1

Correlations between DMM Capacity, Asymptotic Ability, and Age 20 Longitudinal IRT Score with 95\% confidence intervals in parentheses for Michaelis-Menten trajectory

\begin{tabular}{|c|c|c|c|c|c|}
\hline & & \multicolumn{2}{|c|}{ Pearson } & \multicolumn{2}{|c|}{ Spearman } \\
\hline & & $\begin{array}{c}\text { With } \\
\text { Outliers }\end{array}$ & $\begin{array}{l}\text { Without } \\
\text { Outliers }\end{array}$ & $\begin{array}{c}\text { With } \\
\text { Outliers }\end{array}$ & $\begin{array}{l}\text { Without } \\
\text { Outliers }\end{array}$ \\
\hline Asymptotic Ability & DMM Capacity & $\begin{array}{c}.69 \\
(.62, .74)\end{array}$ & $\begin{array}{c}.70 \\
(.64, .76)\end{array}$ & $\begin{array}{c}.67 \\
(.60, .73)\end{array}$ & $\begin{array}{c}.68 \\
(.61, .74)\end{array}$ \\
\hline Asymptotic Ability & Age 20 Longitudinal IRT Score & $\begin{array}{c}.40 \\
(.30, .49)\end{array}$ & $\begin{array}{c}.46 \\
(.36, .55)\end{array}$ & $\begin{array}{c}.48 \\
(.38, .56)\end{array}$ & $\begin{array}{c}.49 \\
(.39, .57)\end{array}$ \\
\hline \multirow[t]{2}{*}{ DMM Capacity } & Age 20 Longitudinal IRT Score & $\begin{array}{c}.54 \\
(.45, .62) \\
\end{array}$ & $\begin{array}{c}.55 \\
(.47, .63) \\
\end{array}$ & $\begin{array}{c}.61 \\
(.53, .68) \\
\end{array}$ & $\begin{array}{c}.60 \\
(.39, .57) \\
\end{array}$ \\
\hline & & $N=280$ & $N=277$ & $N=280$ & $N=277$ \\
\hline
\end{tabular}


Table A2

$R^{2}$ variance explained measures for a linear regression of Asymptotic Ability on DMM Capacity or Age 20 Longitudinal IRT Score with $95 \%$ confidence interval in parentheses

\begin{tabular}{cccc}
\hline Outcome & Predictor & $\begin{array}{c}R^{2} \\
\text { With Outliers }\end{array}$ & $\begin{array}{c}R^{2} \\
\text { Without Outliers }\end{array}$ \\
\hline Asymptotic Ability & DMM Capacity & $\begin{array}{c}47 \% \\
(39 \%, 55 \%)\end{array}$ & $\begin{array}{c}50 \% \\
(42 \%, 58 \%)\end{array}$ \\
& Last Observed Ability & $\begin{array}{c}16 \% \\
(8 \%, 24 \%)\end{array}$ & $\begin{array}{c}21 \% \\
(12 \%, 30 \%)\end{array}$ \\
\hline \multicolumn{2}{c}{$\Delta R^{2}$} & $31 \%$ & $28 \%$ \\
\hline DMM Relative \% Increase & $196 \%$ & $133 \%$ \\
\hline
\end{tabular}

Note: confidence intervals for $R^{2}$ are calculated using the approximation from Olkin and Finn (1995) 

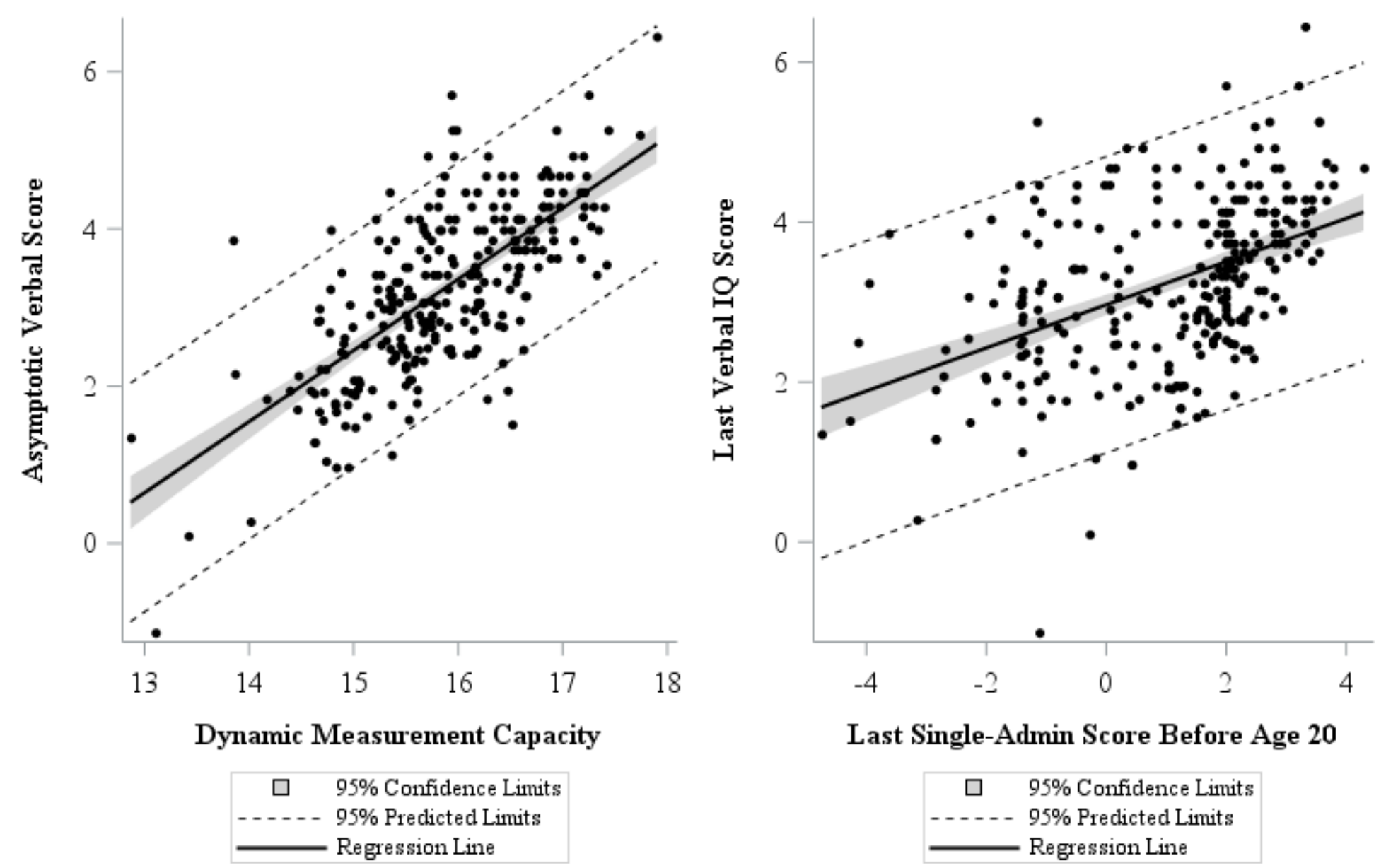

Last Single-Admin Score Before Age 20

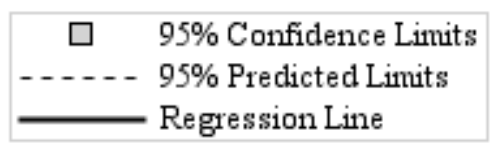

Figure A1. Comparison of fitted regression lines using the Asymptotic Ability in the data as predicted by DMM capacities (left) or Age 20 Longitudinal IRT Score (right) for the data without outliers. The plot on the left has more desirable forecasting qualities $\left(R^{2}=0.50, \mathrm{MSE}=\right.$ $.56)$ compared to the plot on the right $\left(R^{2}=0.21, \mathrm{MSE}=.88\right)$. 


\section{Appendix B: Exponential Trajectory}

\section{SAS Proc Nlmixed Code}

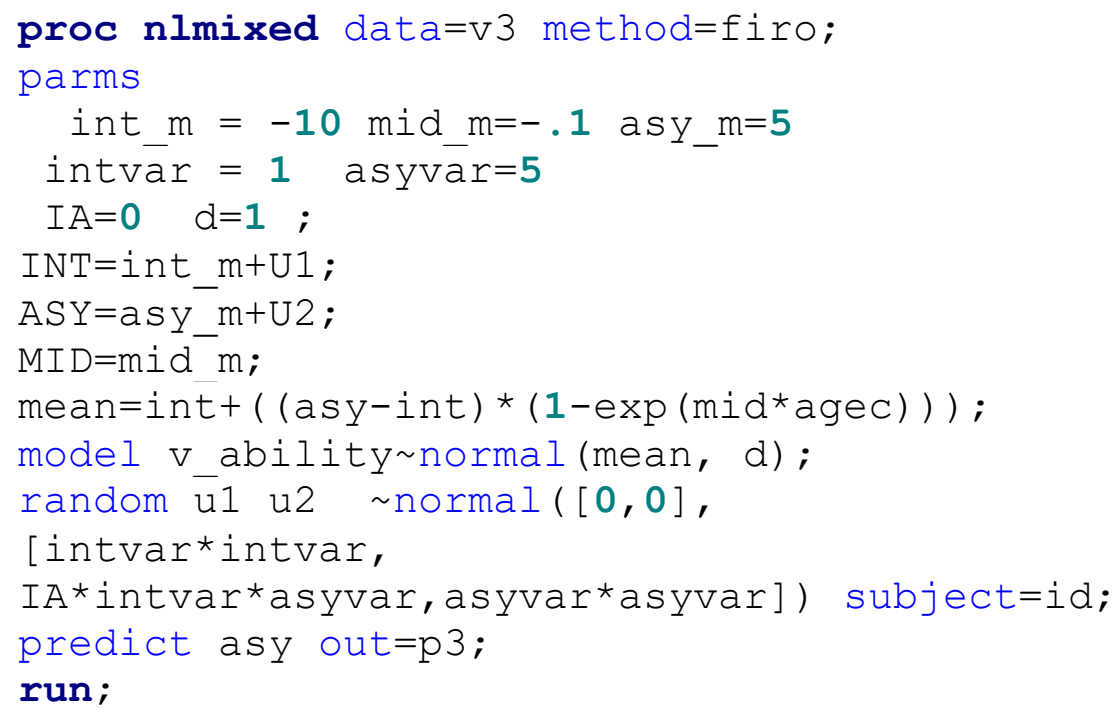

Table B1

Correlations between DMM Capacity, Asymptotic Ability, and Age 20 Longitudinal IRT Score with $95 \%$ confidence intervals in parentheses for Michaelis-Menten trajectory

\begin{tabular}{|c|c|c|c|c|c|}
\hline & & \multicolumn{2}{|c|}{ Pearson } & \multicolumn{2}{|c|}{ Spearman } \\
\hline & & $\begin{array}{c}\text { With } \\
\text { Outliers }\end{array}$ & $\begin{array}{l}\text { Without } \\
\text { Outliers }\end{array}$ & $\begin{array}{c}\text { With } \\
\text { Outliers }\end{array}$ & $\begin{array}{l}\text { Without } \\
\text { Outliers }\end{array}$ \\
\hline Asymptotic Ability & DMM Capacity & $\begin{array}{c}.67 \\
(.60, .73)\end{array}$ & $\begin{array}{c}.69 \\
(.62, .74)\end{array}$ & $\begin{array}{c}.66 \\
(.58, .72)\end{array}$ & $\begin{array}{c}.67 \\
(.59, .73)\end{array}$ \\
\hline Asymptotic Ability & Age 20 Longitudinal IRT Score & $\begin{array}{c}.40 \\
(.30, .49)\end{array}$ & $\begin{array}{c}.46 \\
(.36, .55)\end{array}$ & $\begin{array}{c}.48 \\
(.38, .56)\end{array}$ & $\begin{array}{c}.49 \\
(.39, .57)\end{array}$ \\
\hline \multirow[t]{2}{*}{ DMM Capacity } & Age 20 Longitudinal IRT Score & $\begin{array}{c}.54 \\
(.47, .60) \\
\end{array}$ & $\begin{array}{c}.51 \\
(.44, .57) \\
\end{array}$ & $\begin{array}{c}.55 \\
(.49, .61) \\
\end{array}$ & $\begin{array}{c}.54 \\
(.47, .60) \\
\end{array}$ \\
\hline & & $N=280$ & $N=277$ & $N=280$ & $N=277$ \\
\hline
\end{tabular}


Table B2

$R^{2}$ variance explained measures for a linear regression of Asymptotic Ability on DMM Capacity or Age 20 Longitudinal IRT Score with 95\% confidence interval in parentheses

\begin{tabular}{cccc}
\hline Outcome & Predictor & $\begin{array}{c}R^{2} \\
\text { With Outliers }\end{array}$ & $\begin{array}{c}R^{2} \\
\text { Without Outliers }\end{array}$ \\
\hline Asymptotic Ability & DMM Capacity & $\begin{array}{c}45 \% \\
(36 \%, 54 \%)\end{array}$ & $\begin{array}{c}47 \% \\
(39 \%, 56 \%)\end{array}$ \\
& Last Observed Ability & $\begin{array}{c}16 \% \\
(8 \%, 24 \%)\end{array}$ & $\begin{array}{c}21 \% \\
(12 \%, 30 \%)\end{array}$ \\
\hline \multicolumn{2}{c}{$\Delta R^{2}$} & $29 \%$ & $26 \%$ \\
\hline DMM Relative \% Increase & $181 \%$ & $124 \%$ \\
\hline
\end{tabular}

Note: confidence intervals for $R^{2}$ are calculated using the approximation from Olkin and Finn (1995) 


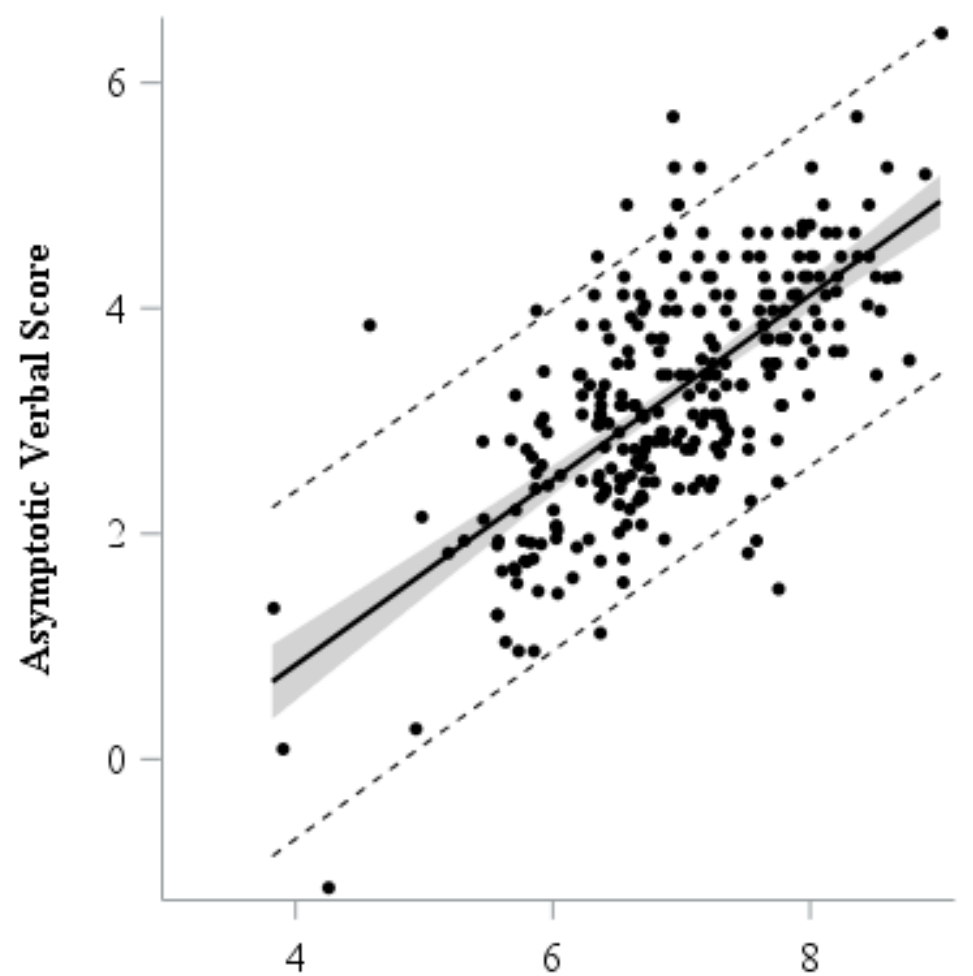

Dynamic Measurement Capacity

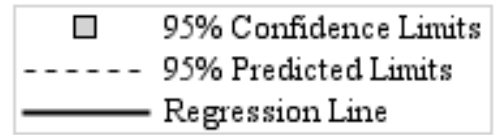

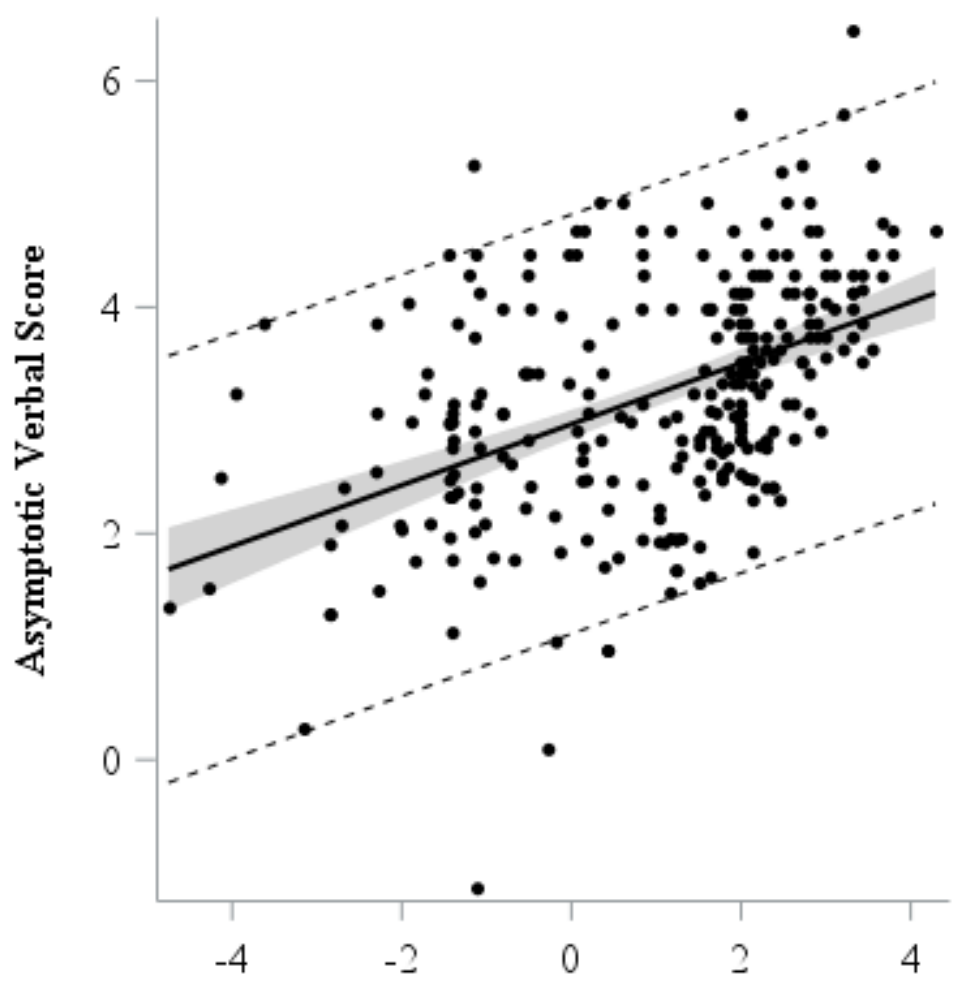

Age 20 Longitudinal IRT Score

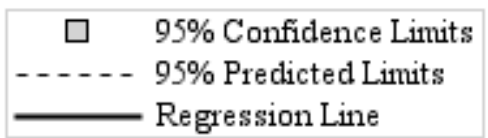

Figure B1. Comparison of fitted regression lines using the Asymptotic Ability in the data as predicted by DMM capacities (left) or Age 20 Longitudinal IRT Score (right) for the data without outliers. The plot on the left has more desirable forecasting qualities $\left(R^{2}=0.47, \mathrm{MSE}=\right.$ $.59)$ compared to the plot on the right $\left(R^{2}=0.21, \mathrm{MSE}=.88\right)$. 


\section{Appendix C: Weibull Trajectory}

\section{SAS Proc Nlmixed Code}

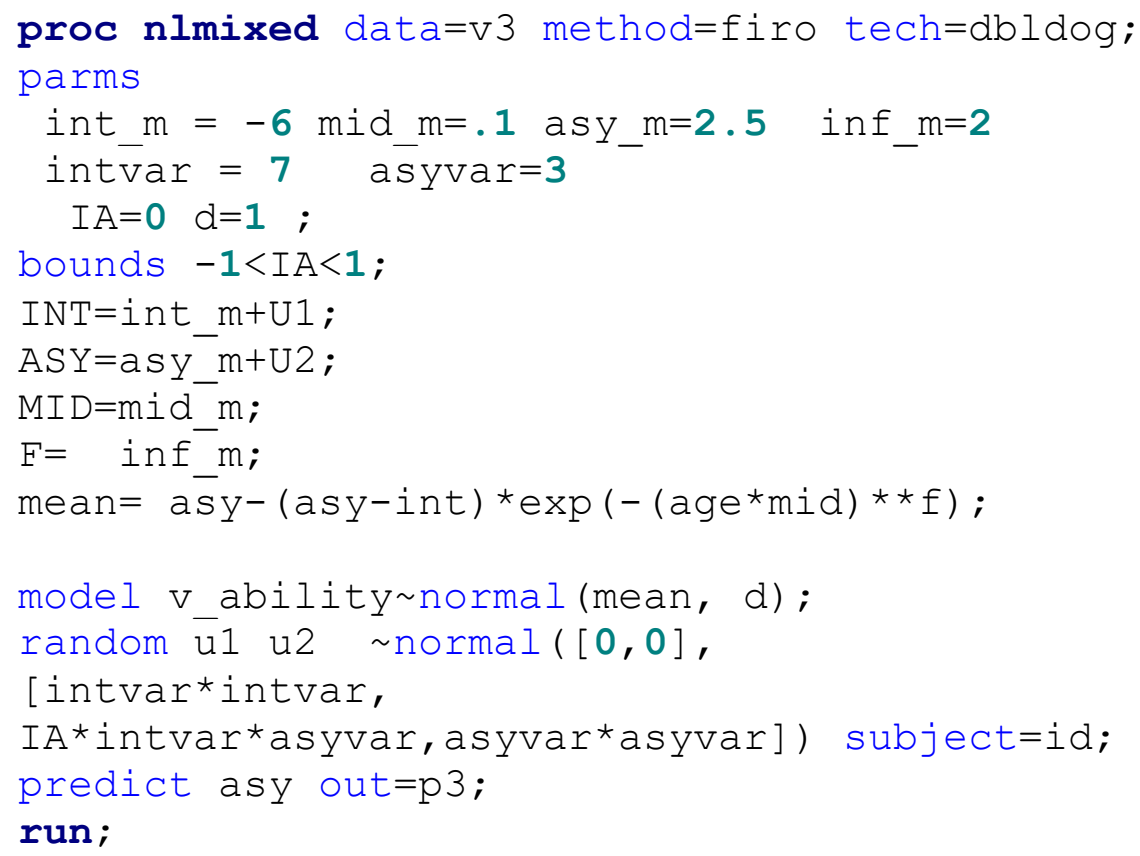

Table C1

Correlations between DMM Capacity, Asymptotic Ability, and Age 20 Longitudinal IRT Score with $95 \%$ confidence intervals in parentheses for Michaelis-Menten trajectory

\begin{tabular}{lccccc}
\hline & & \multicolumn{2}{c}{ Pearson } & \multicolumn{2}{c}{ Spearman } \\
\hline & & With & Without & With & Without \\
& & Outliers & Outliers & Outliers & Outliers \\
\hline Asymptotic Ability & DMM Capacity & .66 & .68 & .64 & .66 \\
& & $(.58, .72)$ & $(.61, .74)$ & $(.57, .71)$ & $(.59, .72)$ \\
Asymptotic Ability & Age 20 Longitudinal IRT Score & .40 & .46 & .48 & .49 \\
& & $(.30, .49)$ & $(.36, .55)$ & $(.38, .56)$ & $(.39, .57)$ \\
DMM Capacity & Age 20 Longitudinal IRT Score & .58 & .60 & .60 & .64 \\
& & $(.51, .63)$ & $(.52, .67)$ & $(.54, .65)$ & $(.56, .70)$ \\
\hline
\end{tabular}


Table C2

$R^{2}$ variance explained measures for a linear regression of Asymptotic Ability on DMM Capacity or Age 20 Longitudinal IRT Score with $95 \%$ confidence interval in parentheses

\begin{tabular}{cccc}
\hline Outcome & Predictor & $R^{2}$ & $R^{2}$ \\
With Outliers & Without Outliers \\
\hline Asymptotic Ability & DMM Capacity & $\begin{array}{c}43 \% \\
(34 \%, 52 \%)\end{array}$ & $\begin{array}{c}46 \% \\
(38 \%, 54 \%)\end{array}$ \\
& Last Observed Ability & $\begin{array}{c}16 \% \\
(8 \%, 24 \%)\end{array}$ & $\begin{array}{c}21 \% \\
(12 \%, 30 \%)\end{array}$ \\
\hline \multicolumn{2}{c}{$\Delta R^{2}$} & $27 \%$ & $25 \%$ \\
\hline DMM Relative \% Increase & $169 \%$ & $119 \%$ \\
\hline
\end{tabular}

Note: confidence intervals for $R^{2}$ are calculated using the approximation from Olkin and Finn (1995) 


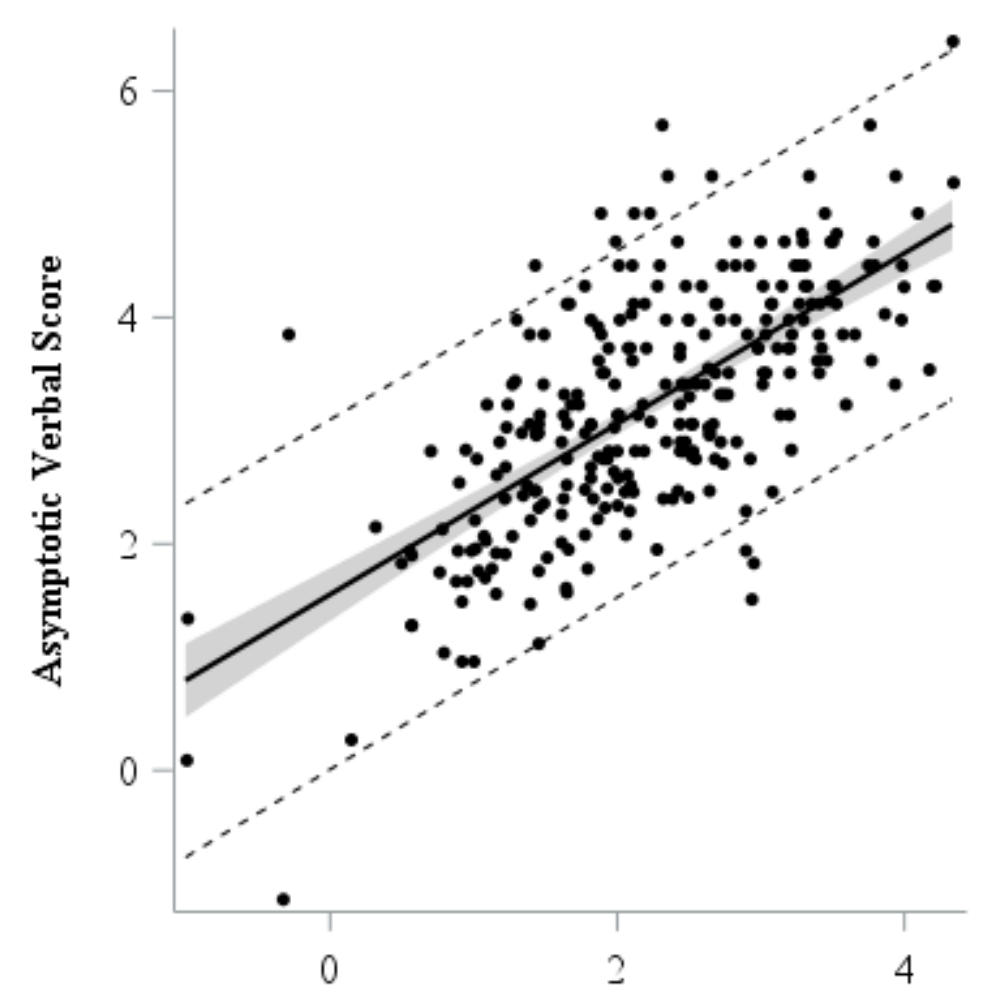

Dynamic Measurement Capacity

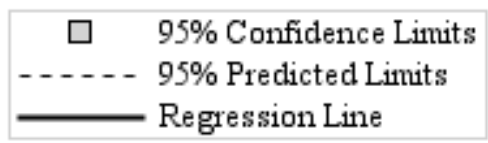

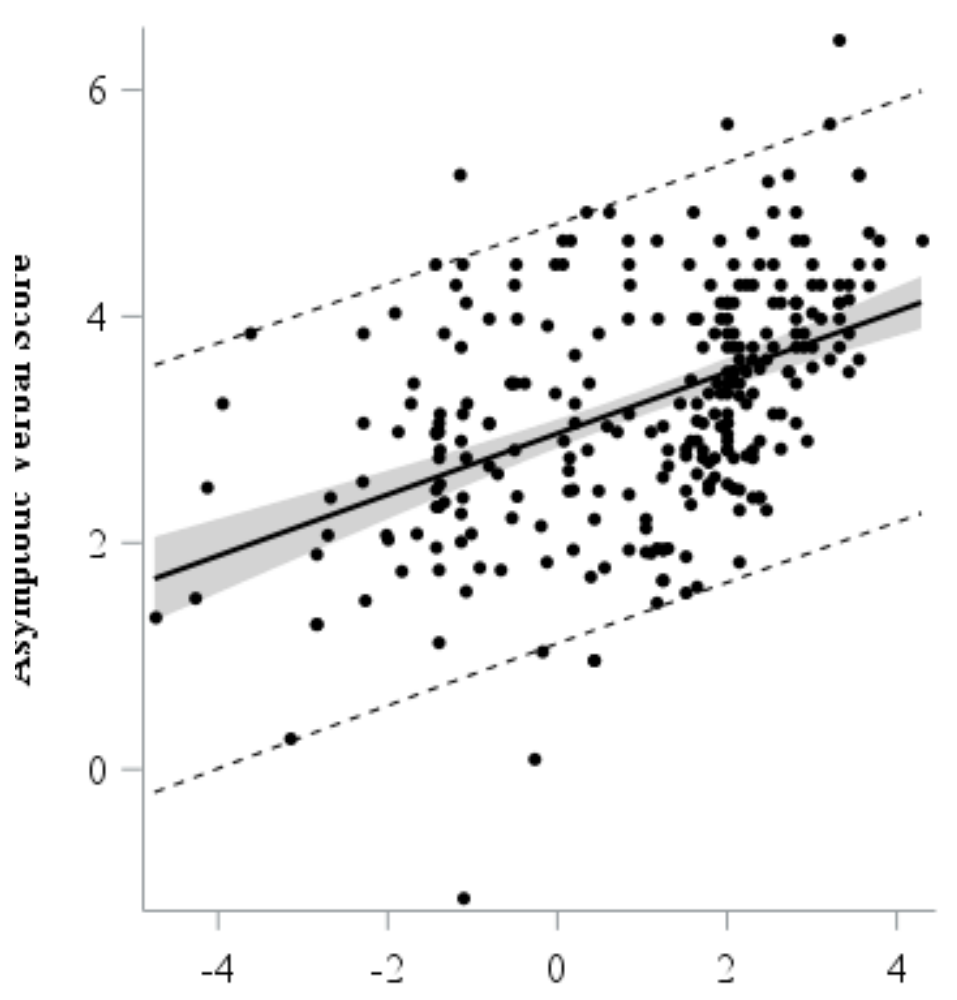

Age 20 Longitudinal IRT Score

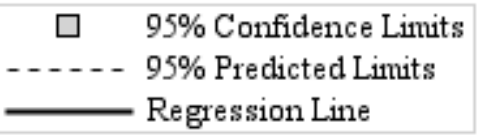

Figure C1. Comparison of fitted regression lines using the Asymptotic Ability in the data as predicted by DMM capacities (left) or Age 20 Longitudinal IRT Score (right) for the data without outliers. The plot on the left has more desirable forecasting qualities $\left(R^{2}=0.46, \mathrm{MSE}=\right.$ $.60)$ compared to the plot on the right $\left(R^{2}=0.21, \mathrm{MSE}=.88\right)$. 


\section{Appendix D: Sensitivity Analysis Tables}

Table D1

Correlations between DMM Capacity, Asymptotic Ability, and Age 12 Longitudinal IRT Score with $95 \%$ confidence intervals in parentheses based on an age 12 cut-off

\begin{tabular}{lcccccc}
\hline & & \multicolumn{2}{c}{ Pearson } & \multicolumn{2}{c}{ Spearman } \\
\hline & & $\begin{array}{c}\text { With } \\
\text { Outliers }\end{array}$ & $\begin{array}{c}\text { Without } \\
\text { Outliers }\end{array}$ & $\begin{array}{c}\text { With } \\
\text { Outliers }\end{array}$ & $\begin{array}{c}\text { Without } \\
\text { Outliers }\end{array}$ \\
\hline Asymptotic Ability & DMM Capacity & .61 & .62 & .59 & .60 \\
& & $(.53, .68)$ & $(.54, .69)$ & $(.51, .67)$ & $(.52, .67)$ \\
Asymptotic Ability & Age 12 Longitudinal IRT Score & .50 & .55 & .52 & .53 \\
& & $(.40, .58)$ & $(.46, .63)$ & $(.43, .60)$ & $(.43, .61)$ \\
& & & & & & \\
DMM Capacity & Age 12 Longitudinal IRT Score & .77 & .81 & .77 & .80 \\
& & & $(.73, .80)$ & $(.77, .85)$ & $(.73, .81)$ & $(.75, .84)$ \\
\hline
\end{tabular}

Table D2

Correlations between DMM Capacity, Asymptotic Ability, and Age 15 Longitudinal IRT Score with $95 \%$ confidence intervals in parentheses based on an age 15 cut-off

\begin{tabular}{|c|c|c|c|c|c|}
\hline & & \multicolumn{2}{|c|}{ Pearson } & \multicolumn{2}{|c|}{ Spearman } \\
\hline & & $\begin{array}{c}\text { With } \\
\text { Outliers }\end{array}$ & $\begin{array}{l}\text { Without } \\
\text { Outliers }\end{array}$ & $\begin{array}{c}\text { With } \\
\text { Outliers }\end{array}$ & $\begin{array}{l}\text { Without } \\
\text { Outliers }\end{array}$ \\
\hline Asymptotic Ability & DMM Capacity & $\begin{array}{c}.63 \\
(.58, .70)\end{array}$ & $\begin{array}{c}.66 \\
(.58, .72)\end{array}$ & $\begin{array}{c}.62 \\
(.54, .69)\end{array}$ & $\begin{array}{c}.64 \\
(.56, .70)\end{array}$ \\
\hline Asymptotic Ability & Age 15 Longitudinal IRT Score & $\begin{array}{c}.48 \\
(.38, .56)\end{array}$ & $\begin{array}{c}.54 \\
(.45, .62)\end{array}$ & $\begin{array}{c}.54 \\
(.45, .62)\end{array}$ & $\begin{array}{c}.55 \\
(.46, .62)\end{array}$ \\
\hline \multirow[t]{2}{*}{ DMM Capacity } & Age 15 Longitudinal IRT Score & $\begin{array}{c}.73 \\
(.68, .77) \\
\end{array}$ & $\begin{array}{c}.78 \\
(.73, .82) \\
\end{array}$ & $\begin{array}{c}.74 \\
(.69, .77) \\
\end{array}$ & $\begin{array}{c}.79 \\
(.73, .83) \\
\end{array}$ \\
\hline & & $N=280$ & $N=277$ & $N=280$ & $N=277$ \\
\hline
\end{tabular}


Table D2

Correlations between DMM Capacity, Asymptotic Ability, and Age 30 Longitudinal IRT Score with $95 \%$ confidence intervals in parentheses based on an age 30 cut-off

\begin{tabular}{lcccccc}
\hline & & \multicolumn{2}{c}{ Pearson } & \multicolumn{2}{c}{ Spearman } \\
\hline & & $\begin{array}{c}\text { With } \\
\text { Outliers }\end{array}$ & $\begin{array}{c}\text { Without } \\
\text { Outliers }\end{array}$ & $\begin{array}{c}\text { With } \\
\text { Outliers }\end{array}$ & $\begin{array}{c}\text { Without } \\
\text { Outliers }\end{array}$ \\
\hline Asymptotic Ability & DMM Capacity & .67 & .69 & .65 & .67 \\
& & $(.59, .73)$ & $(.62, .75)$ & $(.58, .72)$ & $(.60, .73)$ \\
Asymptotic Ability & Age 30 Longitudinal IRT Score & .40 & .46 & .47 & .48 \\
& & $(.30, .50)$ & $(.37, .55)$ & $(.38, .56)$ & $(.39, .57)$ \\
& & & & & & \\
DMM Capacity & Age 30 Longitudinal IRT Score & .59 & .61 & .61 & .65 \\
& & & $(.52, .64)$ & $(.53, .68)$ & $(.55, .66)$ & $(.57, .71)$ \\
\hline
\end{tabular}




\section{Appendix E: Logistic Model with Cholesky Decomposition}

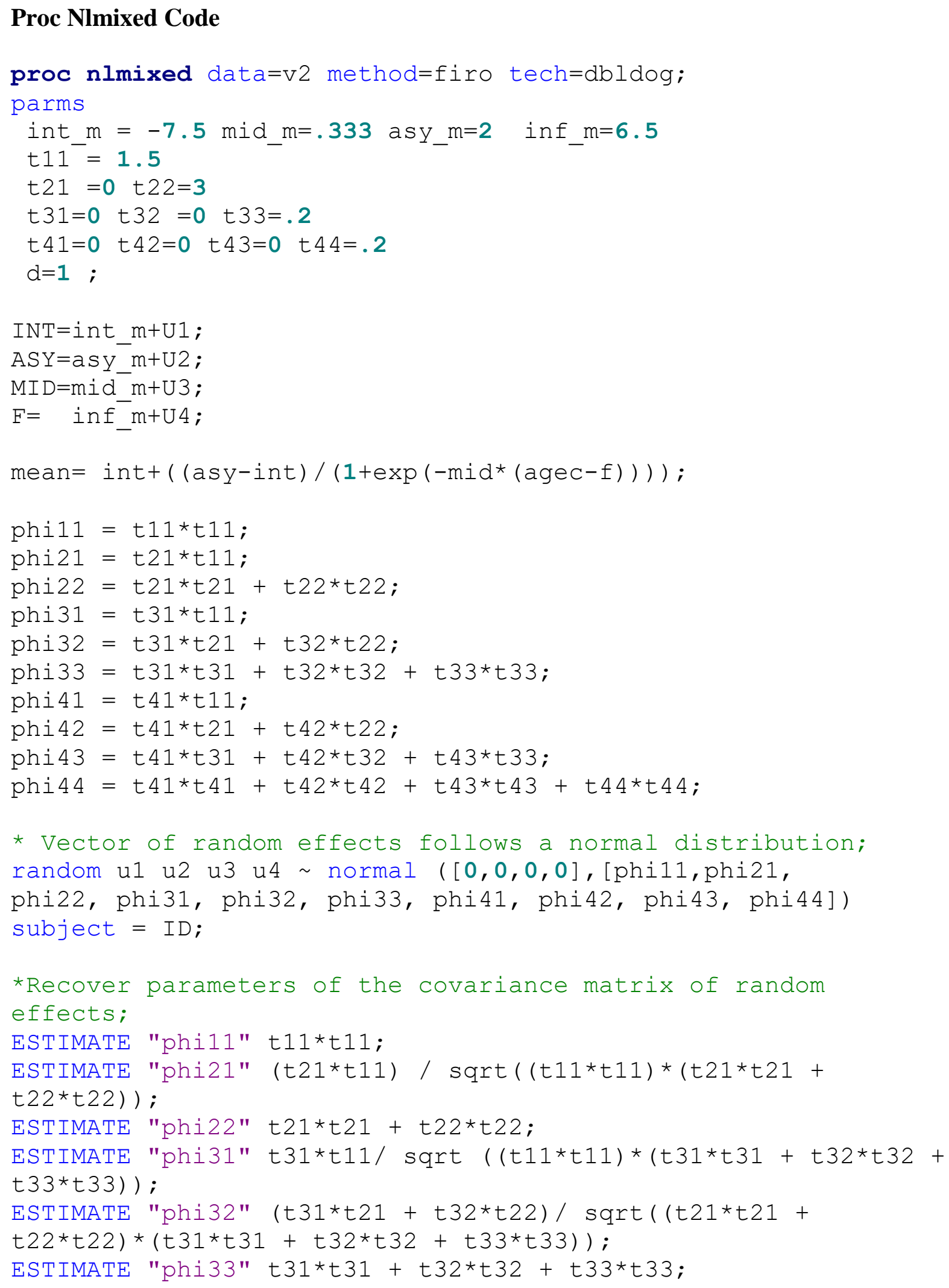




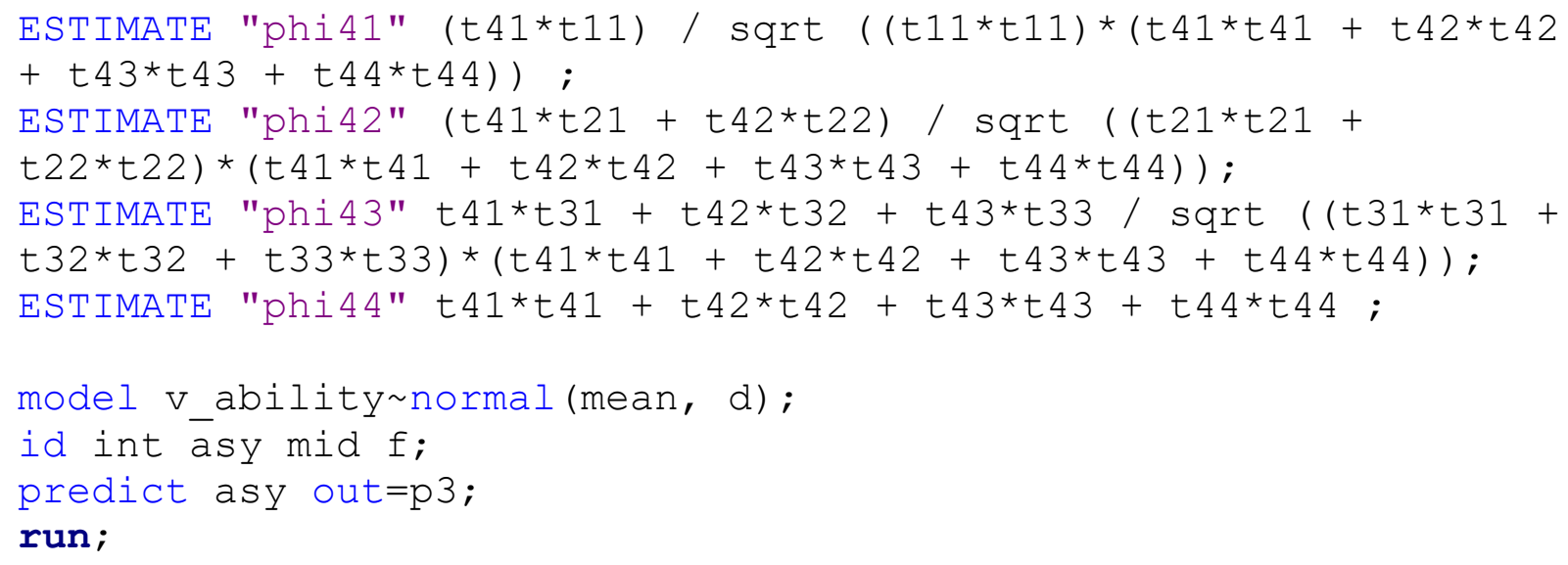

Table E1

Parameter estimates for logistic model parameterized with Cholesky decomposition for the random effect covariance matrix

\begin{tabular}{|c|c|c|}
\hline Parameter & Notation & Estimate \\
\hline \multicolumn{3}{|c|}{ Fixed Effects } \\
\hline Capacity & $\gamma_{C}$ & 2.22 \\
\hline Intercept & $\gamma_{0}$ & -6.98 \\
\hline Rate & $\gamma_{R}$ & .40 \\
\hline Midpoint & $\gamma_{M}$ & 6.73 \\
\hline \multicolumn{3}{|c|}{ Random Effect Variances } \\
\hline Capacity & $\tau_{C}$ & .348 \\
\hline Intercept & $\tau_{0}$ & 2.30 \\
\hline Rate & $\tau_{R}$ & .001 \\
\hline Midpoint & $\tau_{M}$ & 2.21 \\
\hline \multicolumn{3}{|c|}{ Random Effect Correlations } \\
\hline Intercept, Capacity & $\operatorname{Corr}\left(u_{0}, u_{C}\right)$ & -.11 \\
\hline Intercept, Rate & $\operatorname{Corr}\left(u_{0}, u_{R}\right)$ & -.05 \\
\hline Intercept, Midpoint & $\operatorname{Corr}\left(u_{0}, u_{M}\right)$ & -.91 \\
\hline Capacity, Rate & $\operatorname{Corr}\left(u_{C}, u_{R}\right)$ & .99 \\
\hline Capacity, Midpoint & $\operatorname{Corr}\left(u_{C}, u_{M}\right)$ & .52 \\
\hline Rate, Midpoint & $\operatorname{Corr}\left(u_{R}, u_{M}\right)$ & .03 \\
\hline \multicolumn{2}{|l|}{$\mathrm{BIC}$} & 5,537 \\
\hline
\end{tabular}


Table E2

Correlations between DMM Capacity, Asymptotic Ability, and Age 20 Longitudinal IRT Score with $95 \%$ confidence intervals in parentheses for Michaelis-Menten trajectory

\begin{tabular}{lcccccc}
\hline & & \multicolumn{2}{c}{ Pearson } & \multicolumn{2}{c}{ Spearman } \\
\hline & & With & Without & With & Without \\
& & Outliers & Outliers & Outliers & Outliers \\
\hline Asymptotic Ability & DMM Capacity & .66 & .68 & .64 & .65 \\
& & $(.59, .72)$ & $(.61, .74)$ & $(.57, .71)$ & $(.58, .71)$ \\
Asymptotic Ability & Age 20 Longitudinal IRT Score & .40 & .46 & .48 & .49 \\
& & $(.30, .49)$ & $(.36, .55)$ & $(.38, .56)$ & $(.39, .57)$ \\
& & & & & \\
DMM Capacity & Age 20 Longitudinal IRT Score & .57 & .58 & .63 & .63 \\
& & & $(.49, .64)$ & $(.50, .65)$ & $(.55, .70)$ & $(.55, .69)$ \\
\hline
\end{tabular}


Table E3

$R^{2}$ variance explained measures for a linear regression of Asymptotic Ability on DMM Capacity or Age 20 Longitudinal IRT Score with $95 \%$ confidence interval in parentheses

\begin{tabular}{cccc}
\hline Outcome & Predictor & $R^{2}$ & $R^{2}$ \\
With Outliers & Without Outliers \\
\hline Asymptotic Ability & DMM Capacity & $\begin{array}{c}44 \% \\
(36 \%, 53 \%)\end{array}$ & $\begin{array}{c}46 \% \\
(38 \%, 55 \%)\end{array}$ \\
& Last Observed Ability & $\begin{array}{c}16 \% \\
(8 \%, 24 \%)\end{array}$ & $\begin{array}{c}21 \% \\
(12 \%, 30 \%)\end{array}$ \\
\hline \multicolumn{2}{c}{$\Delta R^{2}$} & $28 \%$ & $25 \%$ \\
\hline DMM Relative \% Increase & $175 \%$ & $119 \%$ \\
\hline
\end{tabular}

Note: confidence intervals for $R^{2}$ are calculated using the approximation from Olkin and Finn (1995) 


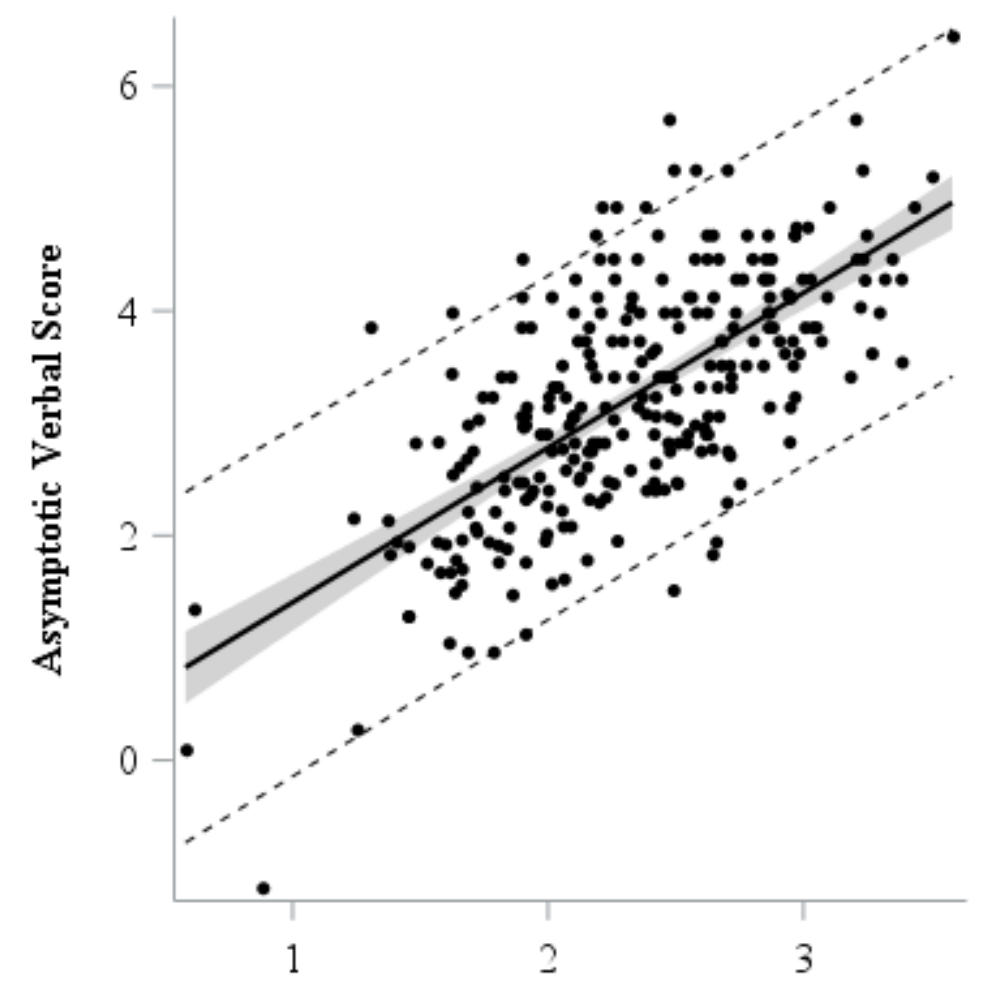

Dynamic Measurement Capacity

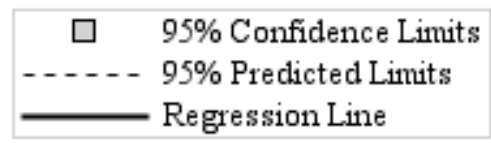

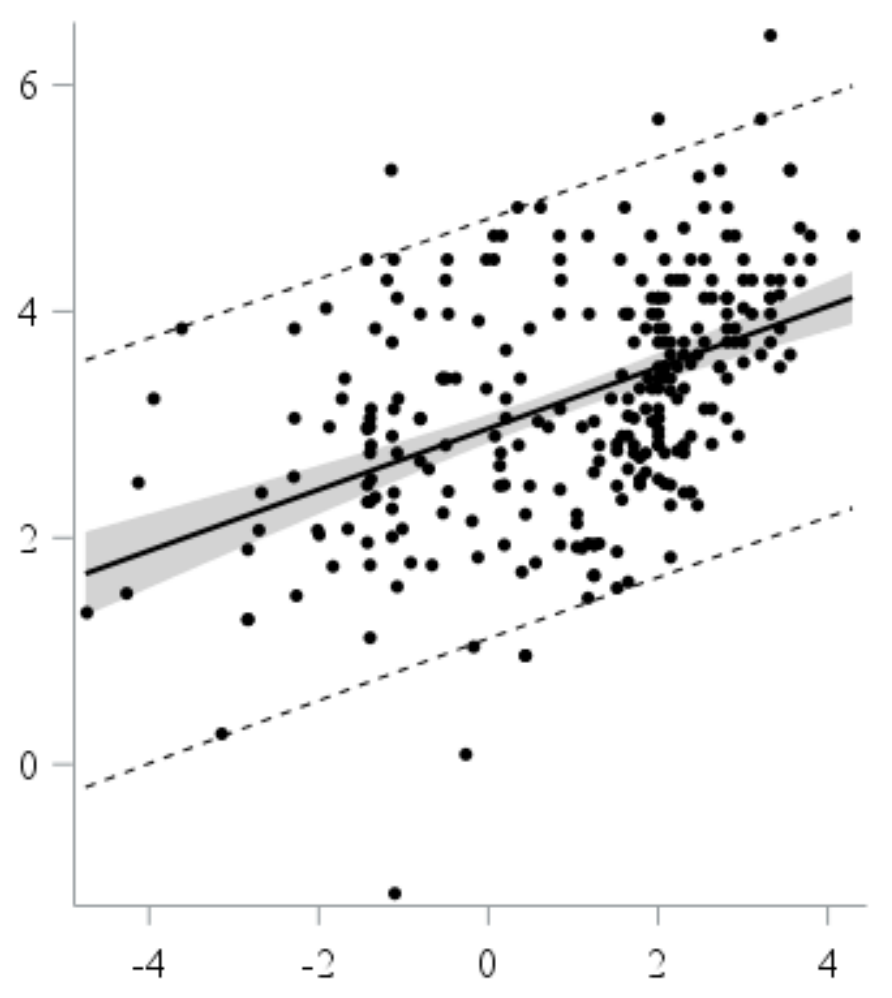

Age 20 Longitudinal IRT Score

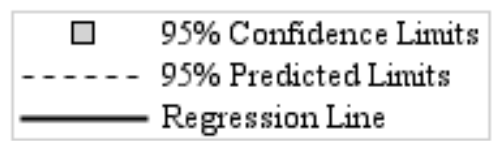

Figure E1. Comparison of fitted regression lines using the Asymptotic Ability in the data as predicted by DMM capacities (left) or Age 20 Longitudinal IRT Score (right) for the data without outliers. The plot on the left has more desirable forecasting qualities $\left(R^{2}=0.46, \mathrm{MSE}=\right.$ $.60)$ compared to the plot on the right $\left(R^{2}=0.21, \mathrm{MSE}=.88\right)$. 


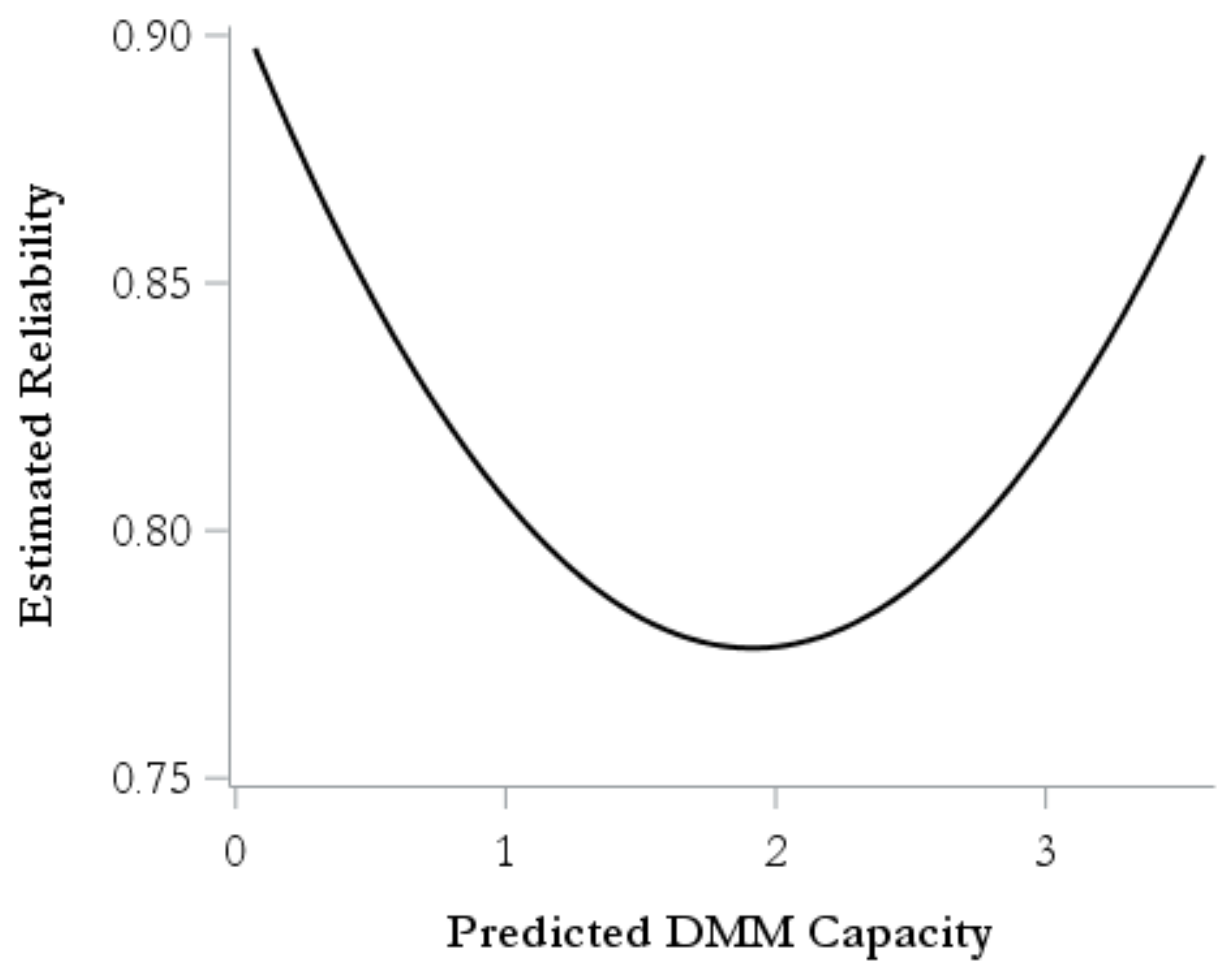

Figure E2. Smoothed conditional reliability plot for the estimated DMM capacities from the logistic model. 